Publ. RIMS, Kyoto Univ.

12 Suppl. (1977), 309-341.

\title{
Quantum Field Theory in Terms of Fourier Hyperfunctions
}

\author{
by \\ Shigeaki NAgAmaCHI* and Nobumichi MUgIBAYASHI**
}

\begin{abstract}
The Wightman axioms are extended to the quantum field theory in terms of Fourier hyperfunctions. The support concept of hyperfunctions is crucial for the formulation of locality and spectral condition. The complete equivalence is proved between modified Wightman axioms for relativistic theory and modified Osterwalder-Schrader axioms for Euclidean theory.
\end{abstract}

\section{§ 1. Introduction}

The aim of the present series of papers is to extend as far as possible the framework of the axiomatic quantum field theory and at the same time to establish the axioms for Euclidean Green's functions which are completely equivalent to a set of axioms for the relativistic vacuum expectations (modified Wightman axioms). We have shown in previous papers $[11,12]$ that this aim is achieved on the basis of the theory of Fourier hyperfunctions which was founded by M. Sato, in place of tempered distributions playing a central role in Wightman's original formulation of the axiomatic quantum field theory.

Several authors have attempted to extend the Wightman axioms for quantum field theory so as to include into the theory a wider class of fields which, owing to singular (or nonrenormalizable) interactions, are no longer described by tempered distributions $[1,2,8]$. In the first paper of the present series [11], which will be quoted as NM I, we succeeded to formulate the quantum field theory in terms of Fourier hyperfunctions which had been studied extensively by Kawai [10]. The space of Fourier

Received June 10, 1976.

* Department of Mathematics, Faculty of Engineering, Tokushima University, Tokushima 770.

** Department of Electrical Engineering, Kobe University, Kobe 657. 
hyperfunctions is the dual of the space of rapidly decreasing holomorphic functions. Since the latter space contains no function of compact support in the usual sense, we are forced to modify the statement of the locality axiom. This problem was fortunately resolved thanks to a noticeable notion of the "support of hyperfunctions". This concept has been effectively also for the formulation of the spectral condition. The remaining axioms do not require any essential alteration compared with Wightman's axioms. The quantum field theory in terms of Fourier hyperfunctions has been shown to contain all other extensions of Wightman's formulation which were constructed concretely up to the present.

In the recent development of the Euclidean field theory it had been revealed that the temperedness of fields bring inconvenience in asserting the complete equivalence of the relativistic and Euclidean field theory. In order to get a reconstruction theorem for tempered fields satisfying the usual Wightman axioms, Osterwalder and Schrader [14] were compelled to introduce besides the distribution axiom a technical condition, what they called the linear growth condition, into the axioms for Euclidean Green's functions. It is worth tempting to use hyperfunction fields in place of tempered fields to get the complete equivalence of the relativistic and Euclidean field theories. This approach has once been advocated by Glaser [4]. In our second paper [12], which will be referred to as NM II, we have realized this program. In doing so, however, we found it necessary to make a slight extension of the Fourier hyperfunctions, while preserving all the results obtained in NM I. The new Fourier hyperfunction was named the Fourier hyperfunction of the second type in distinction from the old, the Fourier hyperfunction of the first type, which we had used in NM I and forms a subset of the former. In NM II we proved the complete equivalence in question for the case when Wightman functions are assumed to be Fourier hyperfunctions of the second type for temporal variables, while they are of the first type for spatial variables.

The symmetric treatment of time- and space-variables is recovered in the present paper, though the results in NM II still keep their own significance. Here we use only Fourier hyperfunctions of the second type, so we shall omit in what follows the phrase "of the second type", except 
when we feel a special need of using it. In the fourth section Fourier hyperfunctions of the second type are introduced in a way somewhat different from NM I, II and Ref. [7]. For readers' convenience we shall repeat to express some definitions and statements which are found in NM I and II, however this time all in the language of the second type Fourier hyperfunctions. In the last section the complete equivalence of the Euclidean and relativistic field theories in terms of the second type Fourier hyperfunctions will be proved.

\section{$\S 2$. Notations and Conventions}

2.1. Let $z \in \mathbb{C}^{n}, z=x+i y$, and $x \in \mathbb{R}^{n}, y \in \mathbb{R}^{n}$. We follow the standard notations of $n$-tuple of numbers. Thus, let $k=\left(k_{1}, \cdots, k_{n}\right)$ and $l=\left(l_{1}, \cdots, l_{n}\right)$ be $n$-tuples of nonnegative integers, then $x^{k}=x_{1}{ }^{k_{1}} \cdots x_{n}{ }^{k_{n}}$ and $D^{l}=\partial^{|l|} / \partial x_{1}{ }^{l_{1}} \cdots \partial x_{n}{ }^{l_{n}}$, where $|l|=l_{1}+\cdots+l_{n} . \quad x>a, \quad a \in \mathbb{R}$, means that $x_{j}>a$ for $1 \leqq j \leqq n$. The notation $|z|^{p}$ is used to denote $|z|^{p}=\left|z_{1}\right|^{p}+\cdots$ $+\left|z_{n}\right|^{p}$, in particular $|z|=\left|z_{1}\right|+\cdots+\left|z_{n}\right| . \quad d \lambda$ is the Lebesgue measure on $\mathbb{R}^{2 n}: d \lambda=d x d y=d x_{1} \cdots d x_{n} d y_{1} \cdots d y_{n}$.

2.2. The Lorentz-invariant inner product is introduced in $\mathbb{R}^{4}$ by writing $x \cdot y=x^{0} y^{0}-x \cdot y$ for two four-vectors $x=\left(x^{0}, x\right)$ and $y=\left(y^{0}, y\right)$, where $x$ and $y$ are three-dimensional space-vectors. We use the notation $\underline{x}_{n}$ to signify a set of $n$ four-vectors $\left(x_{1}, \cdots, x_{n}\right)$ and write $d \underline{x}_{n}$ instead of $d x_{1} \cdots d x_{n}$. The set of $n$ four-vectors in reverse order $\left(x_{n}, \cdots, x_{1}\right)$ is denoted by ${ }_{n} \underline{x}$. For any vector $x \in \mathbb{R}^{4}$ it is meant that $\theta x=\left(-x^{0}, x\right)$ and $c x=\left(i x^{0}, x\right)$. This convention also applies to a set of four-vectors $\underline{x}_{n}$ by writing $\theta \underline{x}_{n}=\left(\theta x_{1}, \cdots, \theta x_{n}\right)$ and $\iota \underline{x}_{n}=\left(\iota x_{1}, \cdots, c x_{n}\right)$. On occasion it is more convenient to rebind $\underline{x}_{n}$ in the form $\underline{x}_{n}=\left(\underline{x}_{n}{ }^{0}, \underline{x}_{n}\right)$. Then, rezarding as $\underline{x}_{n}{ }^{0} \in \mathbb{R}^{n}, \underline{\boldsymbol{x}}_{n} \in \boldsymbol{R}^{3 n}$ and $\underline{x}_{n} \in \boldsymbol{R}^{4 n}$, the notations $\left|\underline{x}_{n}{ }^{0}\right|,\left|\underline{\boldsymbol{x}}_{n}\right|$ and $\left|\underline{x}_{n}\right|$ stand for $\sum_{j=1}^{n}\left|x_{j}{ }^{0}\right|, \sum_{j=1}^{n} \sum_{\mu=1}^{3}\left|x_{j}{ }^{\mu}\right|$ and $\sum_{j=1}^{n} \sum_{\mu=0}^{3}\left|x_{j}{ }^{\mu}\right|$, respectively.

2.3. For two sets of four-vectors $\underline{x}_{n}$ and $\underline{y}_{n}$, the notation $((x, y)\rangle_{n}$ is the abbreviation of $\sum_{j=1}^{n}\left(x_{j} \cdot y_{j}\right)$. Similar convention is used for their spatial and temporal components: $\left(\langle\boldsymbol{x}, \boldsymbol{y})_{n}=\sum_{j=1}^{n}\left(\boldsymbol{x}_{j} \cdot y_{j}\right)\right.$ and $\left(\left(x^{0}, \boldsymbol{y}^{0}\right)_{n}\right.$ $=\sum_{j=1}^{n} x_{j}{ }^{0} y_{j}{ }^{0}$. Hence we have $((x, y))_{n}=\left(\left(x^{0}, y^{0}\right)\right\rangle_{n}-((x, y)\rangle_{n}$. Finally, for the 
set of vectors, the difference vectors are defined by $\xi_{0}=x_{1}, \xi_{j}=x_{j+1}-x_{j}$, $1 \leqq j \leqq n-1$, in coordinate space and correspondingly $q_{k}=p_{k+1}+\cdots+p_{n}$, $0 \leqq k \leqq n-1$, in momentum space, so that $\left(\langle p, x)_{n}=q_{0} \cdot \xi_{0}+(\langle q, \xi)\rangle_{n-1}\right.$ holds.

\section{§3. Test Function Spaces}

Let us begin with defining

$$
U_{m}=\{z \in \mathbb{C} ;|\operatorname{Im} z|<(1+|\operatorname{Re} z|) / m\} .
$$

Let further $\mathcal{O}_{c}{ }^{m}\left(U_{m}{ }^{n}\right)$ be a Banach space of those functions which are holomorphic in $U_{m}^{n} \equiv\left[U_{m}\right]^{n}$, continuous in its closure and satisfy

$$
\|f\|_{m} \equiv \sup _{z \in U_{m}^{n}}|f(z)| e^{|z| / m}<\infty .
$$

$\|f\|_{m}$ is the norm of the Banach space $\mathcal{O}_{c}{ }^{m}\left(U_{m}{ }^{n}\right)$. The space of rapidly decreasing holomorphic functions $\mathscr{L}_{n}$ is the inductive limit of the Banach space $\left\{\mathcal{O}_{c}{ }^{m}\left(U_{m}{ }^{n}\right)\right\}: \mathscr{Q}_{n}=\operatorname{ind}_{m} \lim \mathcal{O}_{c}{ }^{m}\left(U_{m}{ }^{n}\right)$. When the dimensionality $n$ is unimportant, we write $\mathscr{L}_{* *}$ instead of $\mathscr{L}_{n}$. $\mathscr{L}_{* *}$ is a DFS-space (a dual Fréchet-Schwartz space) and shown to be nuclear (see, in essential, Ref. [7]).

Lemma 3.1. $\mathscr{L}_{* *}$ is dense in $\mathscr{S}$.

Proof. $C_{0}{ }^{\infty}$ is dense in $\mathscr{S}$. Let $\rho_{\varepsilon}(z)=(\pi \varepsilon)^{-n / 2} \prod_{j=1}^{n} \exp \left(-z_{j}^{2} / 2 \varepsilon\right)$. Then it is clear that $f_{\varepsilon}=\rho_{\varepsilon} * f \in \mathscr{Q}_{* *}$ for any $f \in C_{0}{ }^{\infty}$ and $f_{\varepsilon}$ tends to $f$ in $\mathscr{S}$ as $\varepsilon \rightarrow 0$. This completes the proof.

Proposition 3.2. The Fourier transformation of $\mathscr{L}_{* *}$ is a topological isomorphism.

Proof. It suffices to prove the proposition for the case of one variable. We show that the Fourier transformation is a continuous map from $\mathcal{O}_{c}{ }^{m}\left(U_{m}\right)$ into $\mathcal{O}_{c}{ }^{{ }^{\prime}}\left(U_{m^{\prime}}\right)$ with $m^{\prime}$ such that $m^{\prime 2} /\left(1+m^{\prime}\right)>m$. Let $f(\zeta)$ $\in \mathcal{O}_{c}{ }^{m}\left(U_{m}\right)$ and $z \in U_{m^{\prime}}$, then we have

$$
\begin{aligned}
e^{|z| / m^{\prime}}\left|\int e^{i z \zeta} f(\zeta) d \zeta\right| & \leqq 2 \max _{(+,-)}\left|\int e^{ \pm \varepsilon z} e^{i z \zeta} f(\zeta) d \zeta\right| \\
& \leqq 2 \max _{(+,-)} \int e^{-[x(\eta \mp \varepsilon)+y \xi]}|f(\zeta)| d \zeta
\end{aligned}
$$


where we have written $z=x+i y, \zeta+i \eta$ and $\varepsilon=\left(1+m^{\prime}\right) / m^{\prime 2}$. If the integration is carried out along the path $\eta=\alpha \xi \pm \varepsilon,|\alpha| \leqq 1 / m^{\prime}$, then the above integral converges for $|\alpha x+y| \leqq 1 / m^{\prime}$, i.e. $z \in U_{m^{\prime}}$, since $\mid x(\eta \mp \varepsilon)$ $+y \xi|=| \alpha x+y|| \xi|\leqq| \xi\left|/ m^{\prime} \leqq\right| \xi \mid / m$. Thus we have shown that $\tilde{f}(z)$ $=\int e^{i z \zeta} f(\zeta) d \zeta$ for $z \in U_{m}$, is convergent, analytic and satisfies the inequality

$$
\sup _{z \in U_{m}}|\tilde{f}(z)| e^{|z| / m^{\prime}} \leqq C \sup _{\zeta \in U_{m}}|f(\zeta)| e^{|\zeta| / m} .
$$

The same estimate is valid for the Fourier inverse transformation. The proof is thus completed.

Proposition 3.3. $\mathscr{L}_{n_{1}} \otimes \mathscr{L}_{n_{1}}$ is dense in $\mathscr{L}_{n_{1}+n_{2}}$.

\section{Proof. See NM II.}

In the Euclidean theory we need some classes of distributions, different from those in NM II, whose test function spaces are related to the spaces $\mathscr{S}_{1, m}\left(\mathbb{R}^{n}\right)$ of $\mathbb{C}^{\infty}$ functions satisfying the condition

$$
\|f\|_{m, p} \equiv \sup _{x \in \boldsymbol{R}^{n,|l| \leqq p}}\left|D^{l} f(x)\right| e^{(1 / m)(1-1 / p)|x|}<\infty,
$$

where $p=2,3, \cdots$. The topology of $\mathscr{S}_{1, m}\left(\mathbb{R}^{n}\right)$ is provided by a countable set of norms $\left\{\|\cdot\|_{,, p}\right\}_{p=2}^{\infty}[3]$.

Let $\underline{x}_{n} \in \boldsymbol{R}^{4 n}$ and $\left(\xi_{0}, \underline{\xi}_{n-1}\right)$ be difference vectors, as defined in the preceding section. We introduce subspaces of $\mathscr{S}_{1, m}\left(\mathbb{R}^{4 n}\right)$ as follows:

$$
\begin{gathered}
\mathscr{C}_{0}{ }^{m}\left(\mathbb{R}^{4 n}\right)=\left\{f \in \mathscr{S}_{1, m}\left(\mathbb{R}^{4 n}\right) ; f\left(x_{n}\right)=0\right. \text { if } \\
\left.\left|x_{i}{ }^{0}-x_{j}{ }^{0}\right| \leqq\left(1+\left|\boldsymbol{x}_{i}-\boldsymbol{x}_{j}\right|\right) / m \text { for some } i \neq j\right\}, \\
\mathscr{C}_{<}{ }^{m}\left(\boldsymbol{R}^{4 n}\right)=\left\{f \in \mathscr{S}_{1, m}\left(\mathbb{R}^{4 n}\right) ; f\left(x_{n}\right)=0\right. \text { unless } \\
\left.\xi_{j}^{0}>\left(1+\left|\boldsymbol{\xi}_{j}\right|\right) / m \text { for } 0 \leqq j \leqq n-1\right\}, \\
\mathscr{C}_{+}{ }^{m}\left(\mathbb{R}^{4 n}\right)=\left\{f \in \mathscr{S}_{1, m}\left(\mathbb{R}^{4 n}\right) ; f\left(x_{n}\right)=0\right. \text { unless } \\
\left.x_{j}^{0}>\left(1+\left|\boldsymbol{x}_{j}\right|\right) / m \text { for } 1 \leqq j \leqq n\right\} .
\end{gathered}
$$

Each of these sets equipped with the induced topology of $\mathscr{S}_{1, m}$ is a closed subspace of $\mathscr{S}_{1, m}$. If $m<m^{\prime}$, then $\mathscr{C}_{0}{ }^{m} \subset \mathscr{C}_{0}{ }^{{ }^{\prime}}, \mathscr{C}_{<}{ }^{m} \subset \mathscr{C}_{<}{ }^{m^{\prime}}$ and $\mathscr{C}_{+}{ }^{m}$ $\subset \mathscr{C}_{+}{ }^{m^{\prime}}$. We denote by $\mathscr{C}_{0}, \mathscr{C}_{<}$and $\mathscr{C}_{+}$the inductive limit of $\left\{\mathscr{C}_{0}{ }^{m}\right\}$, $\left\{\mathscr{C}_{<}{ }^{m}\right\}$ and $\left\{\mathscr{C}_{+}{ }^{m}\right\}$. 


\section{§4. Fourier Hyperfunctions}

We compactify $\mathbb{C}^{n}$ by identifying it with $\mathbb{R}^{2 n}$. The compactification of $\boldsymbol{R}^{k}$ is denoted by $\boldsymbol{D}^{k}[10]: \mathbb{D}^{k}=\mathbb{R}^{k}\left\lfloor S_{\infty}{ }^{k-1}\right.$, where $\boldsymbol{S}_{\infty}{ }^{k-1}$ is a $(k-1)$ dimensional sphere at infinity. To each $x \in \mathbb{R}^{k}-\{0\}$ we associate a point $x_{\infty}$ on $S_{\infty}{ }^{k-1}$ such that the point $x$ lies on the ray connecting $x_{\infty}$ and the origin. We identify $S^{k-1}$, a $(k-1)$-dimensional sphere centered at the origin, with $\left[\mathbb{R}^{k}-\{0\}\right] / \boldsymbol{R}_{+}$, where $\boldsymbol{R}_{+}=\{x \in \boldsymbol{R} ; x>0\}$. A natural topology is given to the space $\boldsymbol{D}^{k}$. (i) For $x \in \mathbb{R}^{k}$ a fundamental system of neighbourhoods of $x$ is the set of all open balls containing the point $x$. (ii) For $x \in S_{\infty}{ }^{k-1}$ we write $x=y_{\infty}$ and let $y$ be the corresponding point on $S^{k-1}$. Then a fundamental system of neighborhoods of $x$ is given by $\left\{(C+a) \cup C_{\infty} ; C_{\infty} \ni y_{\infty}\right\}$, where $C$ is an open cone generated by some open neighbourhood of $y$ in $S^{k-1}$ with its vertex at the origin, $a$ is some vector in $\mathbb{R}^{k}$, so that $C+a$ is a cone with its vertex at $a$, and $C_{\infty}$ singnifies the points at infinity of that cone. In what follows we write $Q^{n}=\mathbb{D}^{2 n}$, the compactification of $\mathbb{C}^{n}$.

Definition 4.1. (The sheaf of slowly increasing holomorphic functions). Let $\Omega$ be an open set in $Q^{n}$. We denote by $\widetilde{\widetilde{O}}$ the sheaf determined by a presheaf $\{\widetilde{\mathcal{O}}(\Omega)\}$, where $\widetilde{\mathcal{O}}(\Omega)$ is the set of all holomorphic functions $f(z)\left(\in \mathcal{O}\left(\Omega \cap \mathbb{C}^{n}\right)\right)$ such that $\sup _{z \in K \cap C^{n}}|f(z)| e^{-\varepsilon|z|}<\infty$ for any $\varepsilon>0$ and any compact set $K$ in $\Omega$.

Definition 4.2. (The sheaf of rapidly decreasing holomorphic functions). We denote by $\underset{\approx}{\mathcal{O}}$ the sheaf determined by a presheaf $\{\mathcal{\approx}(\Omega)\}$, where $\mathcal{\approx}(\Omega)$ is the set of all holomorphic functions $f(z)$ $\left(\in \mathcal{O}\left(\Omega \cap C^{n}\right)\right)$ such that for any compact set $K$ in $\Omega$ there exists some positive constant $\delta_{K}$ and the estimate $\sup _{z \in K \cap \boldsymbol{C}^{n}}|f(z)| \exp \left(\delta_{K}|z|\right)<\infty$ holds.

Definition 4.3. (Topology of $\underset{\approx}{\Theta}(K)$ ). Let $K$ be a compact set in $\mathbb{D}^{n}$. We give $\underset{\approx}{\mathcal{O}}(K)$ the inductive limit topology $\operatorname{ind}_{m} \lim \mathcal{O}_{c}{ }^{m}\left(V_{m}\right)$, where $\left\{V_{m}\right\}$ is a fundamental system of neighbourhoods of $K$ in $\boldsymbol{Q}^{n}$, satisfying $V_{m} \supseteq V_{m+1}$, and $\mathcal{O}_{c}{ }^{m}\left(V_{m}\right)$ is the Banach space of all holomorphic functions $f(z) \quad\left(\in \mathcal{O}\left(V_{m} \cap \mathbb{C}^{n}\right)\right)$ that are continuous in $\bar{V}_{m} \cap \mathbb{C}^{n}$ and for 
which $|f(z)| \leqq C e^{-|z| / m}$ holds for some constant $C$ (depending on $f$ ). The norm of $\mathcal{O}_{c}{ }^{m}\left(V_{m}\right)$ is defined by $\|f\|_{m}=\sup _{z \in V_{m} \cap \boldsymbol{C}^{n}}|f(z)| e^{|z| / m}$. With this topology $\underset{\approx}{\mathcal{O}}(K)$ is a DFS-space.

Remark 1. We have used the symbol $V_{m} \supseteq V_{m+1}$ to denote that $V_{m+1}$ has a compact neighbourhood in $V_{m}$ with respect to the topology of $Q^{n}$.

Remark 2. For $K=\mathbb{D}^{n}$ we may construct $V_{m}$ as given by $U_{m}{ }^{n} \cup C_{\infty}$, where $C_{\infty}$ is the points at infinity of $U_{m}{ }^{n}$. Therefore we have $\mathscr{Q}_{* *}$ $=\underset{\approx}{\otimes}\left(\mathbb{D}^{n}\right)$.

Definition 4. 4. Consider a Hilbert space $H$. The $H$-valued Fourier hyperfunction is an element of $L\left(\mathscr{L}_{* *}, H\right)$, where $L\left(\mathscr{L}_{* *}, H\right)$ is the space of all continuous linear operators from $\mathscr{L}_{* *}$ to $H$ equipped with the topology of bounded convergence. In particular if one takes $C$ as $H$ this defines the scalar Fourier hyperfunctions $\left(\mathscr{L}_{* *}\right)^{\prime}$.

Remark. Since $\mathscr{Q}_{* *}$ is a DFS-space and so complete and barreled, and moreover since it is nuclear, $L\left(\mathscr{L}_{* *}, H\right) \cong\left(\mathscr{L}_{* *}\right)^{\prime} \hat{\otimes} H$ by Proposition 50.5 of Treves [18].

Proposition 4.5. A separately continuous multilinear form $M$ on $\left[\mathscr{L}_{1}\right]^{n}$ uniquely defines a Fourier hyperfunction $\mu \in\left(\mathscr{L}_{n}\right)^{\prime}$ such that $M\left(\phi_{1}, \cdots, \phi_{n}\right)=\mu\left(\phi_{1} \times \cdots \times \phi_{n}\right)$ for $\phi_{j} \in \mathscr{Q}_{1}, 1 \leqq j \leqq n$.

\section{Proof. See NM II.}

Now we are going to formulate the support of the Fourier hyperfunction and the sheaf of Fourier hyperfunctions. For this purpose we need some preparations.

Defimition 4.6. An open set $\Omega$ in $Q^{n}$ is said to be an $\widetilde{\mathcal{O}}$-pseudoconvex domain if it satisfies the condition:

(i) $\sup _{\Omega \cap C^{n}, 1 \leqq j \leqq n}\left\{2\left|\operatorname{Im} z_{j}\right|-\left|\operatorname{Re} z_{j}\right|\right\} \leqq M<\infty$.

(ii) There exists a plurisubharmonic function $\theta(z)$ on $\Omega \cap \mathbb{C}^{n}$ having 
the properties that $\{z ; \theta(z) \leqq c\} \subseteq\left(\Omega \cap C^{n}\right)$ for any $c<\infty$ and $\sup _{L \cap \boldsymbol{C}^{n}} \theta(z) \leqq M_{L}<\infty$ for any $L \Subset \Omega$.

The following theorem ensures that any compact set in $\mathbb{D}^{n}$ has a fundamental system of $\widetilde{\widetilde{O}}$-pseudoconvex neighbourhoods.

Theorem 4.7. Let $K$ be a compact set in $\mathbb{D}^{n}$ and $U$ be an open neighbourhood of $K$ in $Q^{n}$, then one can find an $\widetilde{\widetilde{O}}$-pseudoconvex domain $\Omega$ such that $K \subset \Omega \subset U$.

Proof. Since $K$ is compact in $Q^{n}$, one can find $\left\{G_{k}\right\}$ such that $K \cap \mathbb{C}^{n} \mathbb{C} G=\bigcup_{k=1}^{m} G_{k}$, where $G_{k}$ is a relative compact open set in $\mathbb{C}^{n}$ or an open convex cone in $\mathbb{C}^{n}$ and $G_{k} \Subset U$. Let us define

$$
g_{i}(z)=\max \left\{0,\left[2|\operatorname{Im} z|^{2}-\left|\operatorname{Re}\left(z-z^{(i)}\right)\right|^{2}\right] /\left|\operatorname{Im} z^{(i)}\right|\right\}
$$

and

$$
h_{l}(z)=\max \left\{0, c_{l}+\left[2|\operatorname{Im} z|^{2}-\left|\operatorname{Re}\left(z-a^{(l)}\right)\right|^{2}\right] /\left[1+\left|a^{(l)}\right|^{2}\right]\right\},
$$

where $z^{(i)} \in \partial G \cap \mathbb{C}^{n}, c_{l} \in \mathbb{R}$ and $a^{(l)} \in \mathbb{R}^{n}$. Let $\chi(x) \in C^{\infty}(\boldsymbol{R})$ be a convex monotone increasing function such that $\chi(x)=0$ if $x \leqq 0, \chi(x)>0$ if $x>0$. Further we define

$$
p(z)=\sum_{i} \chi\left(g_{i}(z)\right) \text { and } q(z)=\sum_{l} \chi\left(h_{l}(z)\right) .
$$

By a suitable choice of $z^{(i)}, c_{l}$ and $a^{(l)}$ we may assume that the sum in $(4 \cdot 1)$ is locally finite and $p(z)=q(z)=0$ for $z \in K \subset \mathbb{C}^{n}$. Then the domain $V=\left\{z \in \mathbb{C}^{n} ; p(z)<1, q(z)<1\right\}$ is contained in $G$. Since $p(z)$ and $q(z)$ are nonnegative smooth plurisubbarmonic functions, the function defined by

$$
\theta(z)=\frac{1}{1-p(z)}+\frac{1}{1-q(z)}
$$

is plurisubharmonic in $V$. Moreover $\Omega=\stackrel{\circ}{\bar{V}}$, the interior of the closure of $V$, is an $\widetilde{\mathcal{O}}$-pseudoconvex domain with $\theta(z)$ a plurisubaharmonic function.

Lemma 4.8. Let $\Omega$ be a pseudoconvex domain in $\mathbb{C}^{n}$ and $\psi(z)$ be a plurisubharmonic function defined on $\Omega$. We define 


$$
\begin{aligned}
& X \equiv L_{(0,0)}^{2}\left(\Omega, 4 \log \left(1+|z|^{2}\right)+\psi(z)\right), \\
& Y \equiv L_{(0,1)}^{2}\left(\Omega, 2 \log \left(1+|z|^{2}\right)+\psi(z)\right), \\
& Z \equiv L_{(0,2)}^{2}(\Omega, \psi(z)) .
\end{aligned}
$$

Let further $\bar{\partial}$ be the Cauchy-Riemann operator defined in the sense of distributions. Then the sequence $X \stackrel{\bar{\partial}}{\rightarrow} Y \stackrel{\bar{\partial}}{\rightarrow} Z$ is exact.

Proof. Since it is easily checked that

$$
\sum_{i, j} t_{i} \bar{t}_{j}\left(\partial^{2} / \partial z_{i} \partial \bar{z}_{j}\right) \log \left(1+|z|^{2}\right) \geqq|t|^{2} /\left(1+|z|^{2}\right)^{2},
$$

this lemma follows from Theorem 2.2.1' of Hörmander [6].

Lemma 4.9. Let $K$ be a compact subset of $\mathbb{B}^{n}$, then the colvomology group $H^{1}(K, \underset{\approx}{\mathcal{O}})=0$.

Proof. Since $K$ has a fundamental system of $\widetilde{\widetilde{O}}$-pseudoconvex neighbourhoods by Theorem 4.7 , and since $H^{1}(\Omega, \underset{\approx}{\mathcal{O}})$ for an $\widetilde{\widetilde{O}}$-pseudoconvex domain $\Omega$ is an inductive limit of cohomology groups of covering: $H^{1}(\Omega, \underset{\approx}{\mathcal{O}})=$ ind $\lim _{\left\{\Omega_{j}\right\}} H^{1}\left(\left\{\Omega_{j}\right\}, \underset{\approx}{\mathcal{O}}\right)$, where $\left\{\Omega_{j}\right\}$ satisfies

(i) $\Omega=\cup_{j \in N} \Omega_{j}$

(ii) $\Omega_{j} \cap \mathbb{C}^{n}=V_{j}$ is convex,

it is sufficient for the proof of the lemma to show that the group $H^{1}\left(\left\{\Omega_{i}\right\}\right.$, $\underset{\approx}{()}$ is vanishing.

We denote by $C^{s}\left(Z^{l o c}\left\{V_{j}\right\}\right)$ the set of all cochains $c=\left\{c_{J}\right\}$, where $J=\left(j_{0}, \cdots, j_{s}\right) \in N^{s+1}$, satisfying the conditions

(i) $\bar{\partial} c_{J}=0$ in $V_{J} \equiv V_{j_{0}} \cap \cdots \cap V_{j_{s}}$.

(ii) For any finite subset $M$ of $N^{s-1}$

$$
\sum_{J \in M} \int_{V_{J}}\left|c_{J}\right|^{2} d \lambda<\infty
$$

where $d \lambda$ is the Lebesgue measure on $\mathbb{R}^{2 n}$.

For any cocycle $\left\{d_{i j}\right\}$ in $H^{1}\left(\left\{\Omega_{j}\right\}, \underset{\approx}{\mathcal{O}}\right)$ it may be assumed that $c=\left\{c_{i j}\right.$ $\left.=d_{i j} \prod_{\nu=1}^{n} \cosh \left(\varepsilon z_{\nu}\right)\right\}$ defines an element of $C^{1}\left(Z^{l o c}\left\{V_{j}\right\}\right)$ for some positive $\varepsilon$ and obeys the equation $\delta c=0$, where $\delta$ is the coboundary operators. We denote by $\left\{\chi_{j}\right\}$ the partition of unity subordinate to $\left\{V_{j}\right\}$ and put $b_{i}=\sum_{j} \chi_{j} c_{i j}$. Since $\delta c=0$ we have $\delta b=c$, and hence $\delta \bar{\partial} b=0$ because of 
$\bar{\partial} c=0$. Therefore $\bar{\partial} b$ defines a global section $f$. By the assumption that $\Omega$ is $\tilde{\mathcal{O}}$-pseudoconvex, we can find a certain plurisubharmonic function $\psi(z)$ satisfying

(i) $\sum_{j}\left|\bar{\partial} \chi_{j}\right| \leqq e^{\psi(z)}$

(ii) $\sup _{L} \psi(z) \leqq C_{L}$ for any $L \Subset \Omega$.

By Cauchy's inequality we have

$$
\int_{V_{i}}\left|b_{i}\right|^{2} e^{-\phi(z)} d \lambda \leqq \sum_{j} \int_{V_{i}} \chi_{j}\left|c_{i j}\right|^{2} e^{-\phi(z)} d \lambda
$$

for any continuous function $\phi(z)$. Lemma 4.8 and the existence of $\psi(z)$ imply the existence of some function $u$ such that $\bar{\partial} u=f$ and

$$
\int_{L_{\cap} \boldsymbol{C}^{n}}|u|^{2}\left(1+|z|^{2}\right)^{-4} d \lambda<\infty \text {. }
$$

Put $c_{i}{ }^{\prime}=b_{i}-u \mid V_{i}$, then $\bar{\partial} c^{\prime}=0$ and $\delta c^{\prime}=\delta b=c$. Clearly $c^{\prime} \in C^{0}\left(Z^{\text {loc }}\left\{V_{j}\right\}\right)$ and, on defining $d^{\prime}$ by $\left\{c_{i}{ }^{\prime} / \prod_{\nu=1}^{n} \cosh \left(\varepsilon z_{\nu}\right)\right\}$, we have $\delta d^{\prime}=d$ and $H^{1}\left(\left\{\Omega_{j}\right\}\right.$, $\underset{\approx}{()}=0$.

Lemma 4.10. Let $E$ and $F$ be DFS-spaces and $u$ be a continuous linear mapping from $E$ into $F$. Then the following statements are equivalent.

1) $u$ is a homomorphism.

2) $u$ is of closed range.

Proof. See Grothendieck [5].

Lemma 4.11. Let $K_{1}, K_{2}$ be compact subsets of $\boldsymbol{D}^{n}$, then the mapping $\left(f_{1}, f_{2}\right) \rightarrow f_{1}-f_{2}$, where $f_{1} \in \mathcal{O}\left(K_{1}\right), f_{2} \in \mathcal{\mho}\left(K_{2}\right)$ and $f_{1}-f_{2} \in$ $\underset{\approx}{(}\left(K_{1} \cap K_{2}\right)$, is a surjective homomorphism.

Proof. By the preceding lemma it suffices to show that the mapping is surjective, which however follows immediately from Lemma 4.9 and the exactness of the sequence

$$
\underset{\approx}{\mathcal{Q}}\left(K_{1}\right) \times \underset{\approx}{\mathcal{Q}}\left(K_{2}\right) \rightarrow \underset{\approx}{\mathcal{Q}}\left(K_{1} \cap K_{2}\right) \rightarrow H^{1}\left(K_{1} \cup K_{2}, \underset{\approx}{\mathcal{O}}\right)
$$

Definition 4.12. A Fourier hyperfunction $\mu \in L\left(\mathscr{L}_{* *}, H\right)$ is said to 
be carried by a compact subset $K$ of $\boldsymbol{D}^{n}$, if $\mu$ belongs to $L(\Theta(K), H)$.

Theorem 4.13. Suppose $\mu \in L\left(\mathscr{L}_{* *}, H\right)$, then there exists the smallest compact subset of $\mathbb{D}^{n}$ which carries $\mu$.

Proof. Consider two compact sets $K_{1}$ and $K_{2}$ which carry $\mu$. Let $N$ be the kernel of the mapping $\underset{\approx}{\mathcal{O}}\left(K_{1}\right) \times \underset{\approx}{\mathcal{O}}\left(K_{2}\right) \rightarrow \underset{\approx}{\mathcal{O}}\left(K_{1} \cap K_{2}\right)$. If $\left(f_{1}\right.$, $\left.f_{2}\right) \in N$ there is a function $g \in \mathcal{O}_{(}\left(K_{1} \cup K_{2}\right)$, which is an extension of $f_{1}$ and $f_{2}$, and we have $\langle\mu, g\rangle=\left\langle\mu, f_{i}\right\rangle, i=1,2$. Next, take any $f \in \Theta_{\approx}\left(K_{1}\right.$ $\left.\cap K_{2}\right)$ and write it in the form $f=f_{1}-f_{2}$ with some $f_{i} \in \underset{\mathcal{Q}}{\mathcal{O}}\left(K_{i}\right), i=1,2$. Then a linear mapping $\langle\mu, f\rangle$ is defined on $\underset{\approx}{\mathcal{O}}\left(K_{1} \cap K_{2}\right)$, which is irrespective of the decomposition of $f$. The continuity of the linear form comes from Lemma 4.11.

Let $\left\{K_{i}\right\}_{i \in I}$ be an infinite family of compact sets which carry $\mu$. Put $K=\bigcap_{i \in I} K_{i}$. Then for any neighbourhood $U$ of $K$ there exists a finite subset $J$ of $I$ such that $U \supset \bigcap_{i \in J} K_{i}$. From the definition of the topology of $\underset{\approx}{\mathcal{O}}(K)$ and the above argument follows $\mu \in L(\underset{\approx}{\mathcal{*}}(K), H)$. This completes the proof.

Definition 4.14. We call the smallest compact subset in Theorem 4.13 the support of $\mu$ and denote it by supp $\mu$.

Lemma 4.15. Let $K=\bigcup_{i=1}^{p} K_{i}$ be the union of $p$ compact sets

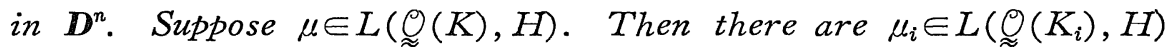
such that $\mu=\sum_{i=1}^{p} \mu_{i}$.

Proof. Since $L(\underset{\approx}{\mathcal{O}}(K), H) \cong\left(\mathcal{\mho}_{(}(K)\right)^{\prime} \hat{\otimes} H$ (see the remark after Definition 4.4), we prove the lemma for the scalar-valued case. The mapping $\underset{\approx}{\mathcal{O}}(K) \rightarrow \prod_{i=1}^{p} \mathcal{\approx}_{\mathcal{*}}\left(K_{i}\right)$, namely $f \rightarrow\left\{f \mid K_{i}\right\}_{i=1}^{p}$, is injective and of closed range, and accordingly the mapping $\prod_{i=1}^{p}\left(\underset{\approx}{(}\left(K_{i}\right)\right)^{\prime} \rightarrow(\underset{\approx}{(}(K))^{\prime}$, namely $\left\{\mu_{i}\right\}_{i=1}^{p} \rightarrow \sum_{i=1}^{p} \mu_{i}$, is surjective.

Definition 4.16 (See Schapira [16]) Let $\Omega$ be an open set in $\boldsymbol{D}^{n}$. We define the space of $H$-valued Fourier hyperfunctions on $\Omega$ by ${ }^{H} \mathscr{R}(\Omega)$ $=L(\underset{\approx}{\Theta}(\bar{\Omega}), H) / L\left(\Theta_{\approx}(\partial \Omega), H\right)$. 
Let $\mu \in L\left(\Theta_{\approx}\left(\mathbb{D}^{n}\right), H\right)$. Because of $\boldsymbol{D}^{n}=\left(\boldsymbol{D}^{n}-\Omega\right) \cup \bar{\Omega}$, we can decompose $\mu=\mu_{1}+\mu_{2}, \mu_{1} \in L\left(\bigotimes_{\approx}\left(\mathbb{D}^{n}-\Omega\right), H\right)$ and $\mu_{2} \in L\left(\bigotimes_{\approx}(\bar{\Omega}), H\right)$ by Lemma 4.15. Therefore it is evident that the canonical mapping

$$
L\left(\bigotimes_{\approx}(\bar{\Omega}), H\right) / L\left(\bigotimes_{\approx}(\partial \Omega), H\right) \rightarrow L\left(\bigotimes_{\approx}\left(\mathbb{D}^{n}\right), H\right) / L\left(\bigotimes_{\approx}\left(\mathbb{D}^{n}-\Omega\right), H\right)
$$

is injective and surjective.

We define the restriction mapping into $\omega \subset \Omega$ by

$$
L\left(\bigotimes_{\approx}\left(\mathbb{D}^{n}\right), H\right) / L\left(\bigotimes_{\approx}\left(\mathbb{D}^{n}-\Omega\right), H\right) \rightarrow L\left(\bigotimes_{\approx}\left(\mathbb{D}^{n}\right), H\right) / L\left(\bigotimes_{\approx}\left(\mathbb{D}^{n}-\omega\right), H\right) .
$$

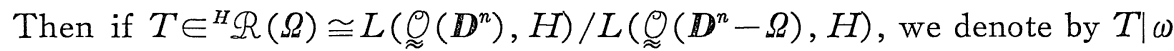
its image in ${ }^{H} \mathscr{R}(\omega)$. It is clear that if $\Omega_{3} \subset \Omega_{2} \subset \Omega_{1}$ and $T \in{ }^{H} \mathscr{R}\left(\Omega_{1}\right)$ then $\left(T \mid \Omega_{2}\right)\left|\Omega_{3}=T\right| \Omega_{3}$. Therefore $\left\{{ }^{H} \mathcal{R}(\Omega)\right\}$ constitutes a presheaf.

\section{Theorem 4.17。}

1) The presheaf $\left\{{ }^{H} R(\Omega)\right\}$ is a sheaf over $\mathbb{D}^{n}$, which is denoted by ${ }^{H} R$.

2) ${ }^{H} \mathscr{R}$ is flabby.

3) If $K$ is a compact set of $\mathbb{D}^{n}$, we have $\Gamma_{K}\left(D^{n},{ }^{H} R\right)=L(\underset{\approx}{(}(K)$, $H)$, where $\Gamma_{K}\left(D^{n},{ }^{H} \mathcal{R}\right)$ is the set of sections whose supports are contained in $K$.

Proof. We here also prove the theorem only for the scalar-valued case.

1a) First we show that if $\Omega=\bigcup_{i \in I} \Omega_{i}$ and $T \in \mathscr{R}(\Omega)$ are such that

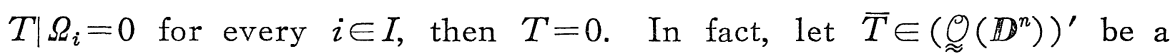

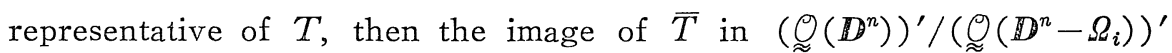
is zero for all $i \in I$. Thus we have supp $\bar{T} \cap \Omega_{i}=\emptyset$ for all $\Omega_{i}$, $i \in I$, and hence supp $\bar{T} \cap \Omega=\emptyset$, which indicates $T=0$.

1b) Let $\Omega=\Omega_{1} \cup \Omega_{2}$ and $T_{i} \in R\left(\Omega_{i}\right), i=1,2$, be such that $T_{1} \mid \Omega_{1} \cap \Omega_{2}$ $=T_{2} \mid \Omega_{1} \cap \Omega_{2} \equiv T$. Their representatives are expressed by $\bar{T} \in\left(\mathcal{\mho}_{\left(\Omega_{1} \cap \Omega_{2}\right)}\right)^{\prime}$ and $\bar{T}_{i} \in\left(\mathcal{\mho}_{\mathcal{O}}\left(\bar{\Omega}_{i}\right)\right)^{\prime}, \quad i=1,2 . \quad$ Then $\operatorname{supp}\left(\bar{T}_{i}-\bar{T}\right) \subset \bar{\Omega}_{i}-\left(\Omega_{1} \cap \Omega_{2}\right)=\bar{\Omega}_{i}$ $\overline{-\left(\Omega_{1} \cap \Omega_{2}\right)} \cup\left(\bar{\Omega}_{i}-\Omega_{i}\right)$. If we define $S_{i}=\bar{T}_{i}-\bar{T}$ and $\bar{T}^{\prime}=\bar{T}+S_{1}+S_{2}$, we see that $\bar{T}^{\prime} \in\left(\underset{\approx}{\left(\Omega_{1} \cup \Omega_{2}\right)}\right)^{\prime}$. The image of $\bar{T}^{\prime}$ in $R\left(\Omega_{1} \cup \Omega_{2}\right)$ will be denoted by $T^{\prime}$. Then we have $T^{\prime} \mid \Omega_{i}=T_{i}$ because supp $\left(T^{\prime}-T_{i}\right) \cap \Omega_{i}$ $=\operatorname{supp} S_{j} \cup \Omega_{i} \subset \overline{\Omega_{j}-\left(\Omega_{1} \cap \Omega_{2}\right)} \cap \Omega_{i}=\emptyset$, in which $j \neq i$.

1c) Let $\Omega=\cup_{i \in I} \Omega_{i}$ and $T_{i} \in R\left(\Omega_{i}\right)$ be such that $T_{i}\left|\Omega_{i} \cap \Omega_{j}=\mathrm{T}_{j}\right| \Omega_{i}$ 
$\cap \Omega_{j}$ for every pair $(i, j)$. One may assume that covering is countable and moreover by the argument $1 \mathrm{~b}$ ) that it is increasing, thus $\Omega=\cup_{n=1}^{\infty} \Omega_{n}$, $\Omega_{n} \subset \Omega_{n+1}, \quad T_{n} \in \mathcal{R}\left(\Omega_{n}\right)$ and $T_{n+p} \mid \Omega_{n}=T_{n}$ for any positive integer $p$. Furthermore it may be assumed that $\bar{\Omega}-\Omega_{n}$ does not have connected components disjointed from $\partial\left(\Omega \cap \boldsymbol{R}^{n}\right)$. Let $\bar{T}_{n} \in\left(\Theta_{\mathcal{N}}\left(\bar{\Omega}_{n}\right)\right)^{\prime}$ be a representative of $T_{n}$ and $d_{n}$ be a distance which provides the topology of $\left(\underset{\approx}{\mathcal{Q}}\left(\bar{\Omega}-\Omega_{n}\right)\right)^{\prime}$. The last assumption on $\bar{\Omega}-\Omega_{n}$ implies that the restriction map $\underset{\approx}{\mathcal{Q}}\left(\bar{\Omega}-\Omega_{n}\right) \rightarrow \underset{\approx}{\mathcal{O}}\left(\partial\left(\Omega \cap \boldsymbol{R}^{n}\right)-\Omega_{n}\right)$ is surjective and accordingly ( $\underset{\approx}{\mathcal{O}}$ $\left.\left(\partial\left(\Omega \cap \boldsymbol{R}^{n}\right)-\Omega_{n}\right)\right)^{\prime}$ is dense in $\left(\mathcal{O}\left(\bar{\Omega}-\Omega_{n}\right)\right)^{\prime}$. Therefore we can take $\phi_{n} \in\left(\left(\partial\left(\Omega \cap \mathbb{R}^{n}\right)-\Omega_{n}\right)\right.$ in such a way that $d_{\nu}\left(\bar{T}_{n+1}-\phi_{n+1}-\left(\bar{T}_{n}-\phi_{n}\right)\right)$ $\leqq 2^{-n}$ for all $\nu \leqq n$. The sequence converges to an element $\bar{T}$ of $(\underset{\widetilde{\sim}}{\mathcal{Q}}(\bar{\Omega}))^{\prime}$ and we have $\bar{T}=\bar{T}-\left(\bar{T}_{n}-\phi_{n}\right)+\left(\bar{T}_{n}-\phi_{n}\right)=\left(\bar{T}_{n}-\phi_{n}\right)+\lim _{p}\left(T_{p}-\phi_{p}\right.$ $\left.-\left(\bar{T}_{n}-\phi_{n}\right)\right)$. Since the sequence $\left\{\bar{T}_{p}-\phi_{p}-\left(\bar{T}_{n}-\phi_{n}\right)\right\}_{p}$ converges in $\left(\underset{\approx}{\mathcal{O}}\left(\bar{\Omega}-\Omega_{n}\right)\right)^{\prime}$, we have $\bar{T}-\left(\bar{T}_{n}-\phi_{n}\right) \in\left(\mathcal{\approx}_{(}\left(\bar{\Omega}-\Omega_{n}\right)\right)^{\prime}$. Therefore $\bar{T} \mid \Omega_{n}$ $=T_{n}$.

2) If $\Omega$ is an open subset of $\mathbb{D}^{n}$ and $T \in R(\Omega)$ there exists a $\bar{T} \in\left(\underset{\widetilde{N}}{\mathcal{O}}\left(\boldsymbol{D}^{n}\right)\right)^{\prime}=\mathscr{R}\left(D^{n}\right)$ such that $\bar{T} \mid \Omega=T$, which shows that the sheaf $\mathcal{R}$ is flabby.

3) Let $K$ be a compact subset of $\mathbb{D}^{n}$. Since $\underset{\approx}{\mathcal{Q}}\left(\mathbb{D}^{n}\right)$ is dense in $\underset{\approx}{\mathcal{O}}(K)$ (see the Appendix), we have an injection $(\underset{\approx}{\mathcal{O}}(K))^{\prime} \rightarrow\left(\underset{\approx}{\mathcal{O}}\left(\mathbb{D}^{n}\right)\right)^{\prime}$. The image of $(\mathcal{\approx}(K))^{\prime}$ is the set of those $T \in\left(\underset{\approx}{(}\left(D^{n}\right)\right)^{\prime}$ which vanish on $\mathbb{D}^{n}-K$, that is, $\Gamma_{K}\left(\mathbb{D}^{n}, R\right)=(\underset{\approx}{(}(K))^{\prime}$.

Remark. ${ }^{H} \mathscr{R}(\Omega)$ can be represented by the cohomology group $H_{\Omega}{ }^{n}\left(V,{ }^{H} \widetilde{\mathcal{O}}\right)$, where $V$ is an open set in $Q^{n}$ which contains $\Omega$ as a relatively closed set. By the excision theorem this representation is independent of the choice of $V$.

Before closing this section we make mention of the Fourier transformation of Fourier hyperfunctions, to which the name of the Fourier hyperfunction owes.

Definition 4.18. For $\mu \in L\left(\mathscr{P}_{* *}, H\right)$ we define its Fourier transform $\mathscr{F} \mu$ by the formula $(\mathscr{F} \mu)(\mathscr{F} f)=\mu(f)$, where

$$
(\mathscr{F} f)(p)=\int e^{i(p, x)} f(x) d x
$$


for $f \in \mathscr{L}_{* *}$.

Since, by Proposition 3.2, the Fourier transformation yields a topological isomorphism of $\mathscr{L}_{* *}$, the definition above is well set up. The sheaf ${ }^{H} \mathscr{B}$ of $H$-valued hyperfunctions over $\boldsymbol{R}^{n}$ coincides with the restriction of the sheaf ${ }^{H} \mathscr{R}$ to $\boldsymbol{R}^{n}$. Hence, because of the flabbiness of the sheaf ${ }^{H} \mathscr{R}$, any $H$-valued Fourier hyperfunction on $\boldsymbol{R}^{n}$ can be extended to an $H$-valued Fourier hyperfunction on $\boldsymbol{D}^{n}$ and one can consider its Fourier transformation.

\section{$\S 5$. Axioms for Fourier Hyperfunction Fields}

In extending the usual Wightman framework of the axiomatic quantum field theory our greatest concern will be how to formulate the locality axiom for extended theory. The strictly localizability of fields $A(f)$ connects intimately with the fact that $f$ belongs to a function space which contains $C^{\infty}$ functions with compact support. Such classes of test functions have been kept more or less in the concrete attempts to extend the Wightman axioms for quantum field theory made so far by several authors $[1,2,8]$. An abstract argument on the class of fields incorporated with the locality in extended sense has been given by Lomsadze and his coworkers [15].

Here we wish to formulate axioms for Fourier hyperfunction fields. Since the test function space $\mathscr{L}_{* *}$ of Fourier hyperfunctions no longer contains any function of compact support, we are obliged to modify the statement of the locality axiom. It will turn out that the celebrated notion of the "support of Fourier hyperfunctions" we have just exhibited in the preceding section plays a crucial role in settling this difficulty and also in the formulation of the spectral condition. Except these the axioms for Fourier hyperfunction fields can be stated in parallel with Wightman's axioms for tempered fields, but for completeness we shall write all of them down mutatis mutandis.

Zeroth Axiom. The space of states is a Hilbert space $H$ over the complex number $C$. For $\Phi, \Psi \in H$ the inner product in $H$ will be denoted by $(\Phi, \Psi)$. 
First Axiom. $\mathscr{Q}_{4}$ is mapped into linear operators $A(f)$ over $H$. $A(f)$ is defined on a dense subset $D$ of $H$, independent of $f \in \mathscr{Q}_{4}$. ( $\Phi$, $A(f) \Psi)$ for $\Phi, \Psi \in D$ is a Fourier hyperfunction and $(\Phi, A(f) \Psi)=$ $(A(\bar{f}) \Phi, \Psi)$. We require that $A(f) D \subset D$.

Second Axiom. A unitary representation $U(a, \Lambda)$ of the restricted Poincaré group exists and satisfies

$$
U(a, \Lambda) A(f) U^{-1}(a, \Lambda)=A\left(f_{\{a, \Lambda\}}\right)
$$

and $U(a, \Lambda) D=D$, where $f_{\{a, \Lambda\}}(x)=f\left(\Lambda^{-1}(x-a)\right)$.

Third Axiom. The spectrum of the energy-momentum operator $P$ is contained in $V_{+}$and there is an invariant state $\Omega$, corresponding to the vacuum, such that $U(a, \Lambda) \Omega=\Omega$. Here $V_{+}=\left\{p=\left(p^{0}, \boldsymbol{p}\right) ; p^{0}>0\right.$, $\left.(p \cdot p) \geqq m^{2}>0\right\} \cup\{p=0\}$. In addition the vacuum $\Omega$ is nondegenerate.

By the first axiom $\left(\Phi, A\left(f_{1}\right) \cdots A\left(f_{n}\right) \Psi\right)$ for $\Phi, \Psi \in D$ is a separately continuous multilinear form on $\left[\mathscr{Q}_{4}\right]^{n}$. Proposition 4.5 asserts that $(\Phi$, $\left.A\left(f_{1}\right) \cdots A\left(f_{n}\right) \Psi\right)$ uniquely determines a Fourier hyperfunction belonging to $\left(\mathscr{L}_{4 n}\right)^{\prime}$ which we denote by $\left(\Phi, A\left(x_{1}\right) \cdots A\left(x_{n}\right) \Psi\right)$ in the sense that formally

$$
\begin{aligned}
& \left(\Phi, A\left(f_{1}\right) \cdots A\left(f_{n}\right) \Psi\right) \\
& \quad=\int\left(\Phi, A\left(x_{1}\right) \cdots A\left(x_{n}\right) \Psi\right) f_{1}\left(x_{1}\right) \cdots f_{n}\left(x_{n}\right) d \underline{x}_{n} .
\end{aligned}
$$

Next for $g\left(\underline{x}_{n}\right)=f_{1}\left(x_{1}\right) \cdots f_{n}\left(x_{n}\right), f_{j} \in \mathscr{P}_{4}$, and $\Phi \in D$ we define an $H$-valued functional $\Phi(g)=A\left(f_{1}\right) \cdots A\left(f_{n}\right) \Phi$ and extend this definition to $\otimes \mathscr{Q}_{4}$ by linearity. By Proposition 3.3, for any $f \in \mathscr{Q}_{4 n}$ there exists a net $\left\{g_{\nu} ; g_{\nu} \in \otimes \mathscr{Q}_{4}\right\}$ such that $g_{\nu} \rightarrow f$ as $\nu \rightarrow \infty$. Therefore $\left\|\Phi_{n}\left(g_{\nu}\right)-\Phi_{n}\left(g_{\mu}\right)\right\|^{2}$ $\rightarrow 0$ as $\nu, \mu \rightarrow \infty$. Thus $\Phi_{n}\left(g_{\nu}\right)$ converges and tends to a vector $\Phi_{n}(f)$ and evidently $\Phi_{n}(f)$ is a continuous linear mapping from $\mathscr{Q}_{4 n}$ to $H$, i.e., $\Phi_{n}(f) \in L\left(\mathscr{Q}_{4 n}, H\right)$. By Definition $4.4 \Phi_{n}(f)$ is an $H$-valued Fourier hyperfunction. We denote it symbolically by $A\left(x_{1}\right) \cdots A\left(x_{n}\right) \Phi$. On the other hand, $\Phi_{n}(f)$ defines a linear operator on $D$ which maps $\Phi$ to $\Phi_{n}(f)$. That operator will be denoted by $\left\langle A^{n}, f\right\rangle$ and written formally as

$$
\left\langle A^{n}, f\right\rangle=\int A\left(x_{1}\right) \cdots A\left(x_{n}\right) f\left(\underline{x}_{n}\right) d \underline{x}_{n} .
$$


The axiom of local commutativity then is formulated as follows:

Fourth Axiom. If $x_{1}$ and $x_{2}$ are spacelike separated, then $A\left(x_{1}\right) A\left(x_{2}\right) \Phi=A\left(x_{2}\right) A\left(x_{1}\right) \Phi$ for every $\Phi \in D$.

Remark. We can restate this axiom in the language of $H$-valued Fourier hyperfunctions by saying that $A\left(x_{1}\right) A\left(x_{2}\right) \Phi-A\left(x_{2}\right) A\left(x_{1}\right) \Phi \in$ $L(\underset{\approx}{\mathcal{O}}(K), H)$ for every $\Phi \in D$, where $K$ is the closure of $\left\{\left(x_{1}, x_{2}\right) \in \boldsymbol{R}^{8}\right.$; $\left.\left(x_{1}-x_{2}\right)^{2}>0\right\}$ in $\mathbb{D}^{8}$.

Fifth Axiom. Let $P(A)$ be an algebra of polynomials in the operators $A(f), f \in \mathscr{Q}_{4}$, then $P(A) \Omega$ is dense in $H$.

Remark. As is evident from Proposition 2.1 of NM I, Proposition 2.3 of NM II and Lemma 3.1, the Fourier hyperfunction fields contain tempered and other existing fields.

\section{§ 6. Modified Wightman Axioms}

\subsection{Wightman Fourier hyperfunctions}

Let $\Omega$ be the vacuum vector. The vacuum expectations $\left(\Omega, A\left(x_{1}\right) \cdots\right.$ $A\left(x_{n}\right) \Omega$ ) are Fourier hyperfunctions which we call Wightman Fourier hyperfunctions ("Wightman functions" for short) and denote by $\mathfrak{W}_{n}\left(\underline{x}_{n}\right)$. From the axioms for Fourier hyperfunction fields stated in the last section, we can deduce various properties of the Wightman functions, which we shall describe in order in the form utilizable also for the modified Wightman axioms.

Fourier hyperfunction property.

$$
\begin{aligned}
\mathfrak{M}_{0}=1, \mathfrak{W}_{n}\left(\underline{x}_{n}\right) \in\left(\mathscr{Q}_{4 n}\right)^{\prime} \quad \text { and } & \\
& \overline{\mathfrak{W}_{n}\left(\underline{x}_{n}\right)}=\mathfrak{M}_{n}\left({ }_{n} \underline{x}\right) \quad \text { for } \quad n \geqq 1 .
\end{aligned}
$$

Relativistic invariance. For each $n, \mathfrak{W}_{n}$ is Poincaré invariant:

$$
\mathfrak{W}_{n}\left(\underline{x}_{n}\right)=\mathfrak{M}_{n}\left(\Lambda \underline{x}_{n}+a\right),
$$


where $\Lambda$ is a proper Lorentz transformation and $\Lambda \underline{x}_{n}+a=\left(\Lambda x_{1}+a, \cdots\right.$, $\left.\Lambda x_{n}+a\right)$.

These two properties are direct consequences of the first and the second axioms, respectively.

Positivity. For any finite sequence of $f_{0}, f_{1}, \cdots, f_{N}$ of test functions such that $f_{0} \in \mathbb{C}, f_{n} \in \mathscr{Q}_{4 n}, 1 \leqq n \leqq N$, there holds the inequality

$$
\sum_{n, m} \mathfrak{N}_{n+m}\left(f_{n}^{*} \times f_{m}\right) \geqq 0
$$

where $\left(f_{n}^{*} \times f_{m}\right)\left(\underline{x}_{n}, \underline{y}_{m}\right)=f_{n}^{*}\left(\underline{x}_{n}\right) f_{m}\left(\underline{y}_{m}\right)$ and $f_{n}^{*}\left(\underline{x}_{n}\right)=\overline{f_{n}\left({ }_{n} \underline{x}\right)}$.

Let $\Omega_{n}\left(f_{n}\right)=\left\langle A^{n}, f_{n}\right\rangle \Omega . \quad$ (R2) is equivalent to $\left\|\sum_{n=0}^{N} \Omega_{n}\left(f_{n}\right)\right\|^{2} \geqq 0$, which follows from the zeroth axiom. The fourth axiom implies

\section{Local commutativity}

$$
\begin{gathered}
\mathfrak{W}_{n}\left(x_{1}, \cdots, x_{j}, x_{j+1}, \cdots, x_{n}\right)=\mathfrak{W}_{n}\left(x_{1}, \cdots, x_{j+1}, x_{j}, \cdots, x_{1}\right) \\
\text { if } \quad\left(x_{j}-x_{j+1}\right)^{2}<0
\end{gathered}
$$

Cluster property. For any space-like vector $a$ and $f \in \mathscr{L}_{4 n}, g \in \mathscr{Q}_{4 m}$ (R4)

$$
\lim _{\lambda \rightarrow \infty} \mathfrak{W}_{n+m}\left(f \times g_{\lambda a}\right)=\mathfrak{W}_{n}(f) \mathfrak{W}_{m}(g),
$$

where $g_{\lambda a}\left(\underline{x}_{m}\right)=g\left(\underline{x}_{m}-\lambda a\right)$. This property comes from the statement in the third axiom that $\{p=0\}$ is an isolated eigenvalue of the energymomentum operator $P$ and the corresponding eigenspace is one-dimensional, as in the case of tempered fields [9].

Spectral condition. By the translational invariance there exist Fourier hyperfunctions $W_{n-1} \in\left(\mathscr{Q}_{4(n-1)}\right)^{\prime}$ such that $\mathfrak{M}_{n}\left(\underline{x}_{n}\right)=W_{n-1}\left(\underline{\xi}_{n-1}\right)$ holds. Then

$$
\operatorname{supp} \widetilde{W}_{n-1}\left(\underline{q}_{n-1}\right) \subset \overline{V_{+}^{n-1}},
$$

where $\overline{V_{+}{ }^{n-1}}$ is the closure of $V_{+}{ }^{n-1}$ in $\mathbb{D}^{4(n-1)}$ and $\widetilde{W}_{n-1}$ is the Fourier transform of $W_{n-1}$. 
Proof of (R5). The proof proceeds as in NM I. When the translation $U(a, 1)$ is applied to $\Omega_{n}(f)=\left\langle A^{n}, f\right\rangle \Omega$ we have by the second axiom (see $\S 2.3$ for notations)

$$
U(a, 1) \Omega_{n}(f)=\Omega_{n}\left(f_{\{a, 1\}}\right)=\widetilde{\Omega}_{n}\left(\tilde{f} e^{i\left(q_{0} \cdot a\right)}\right),
$$

where $\tilde{f}$ is the Fourier transform of $f$. Consider the spectral resolution $U(a, 1)=\int e^{i p . a} d E(p)$ and the integral

$$
\int \chi(p) d E(p) \Omega_{n}(f)=\widetilde{\Omega}_{n}\left(\chi\left(q_{0}\right) \tilde{f}\right)
$$

for any $\chi \in \mathscr{Q}_{4}$. Then mapping $\chi \rightarrow \widetilde{\Omega}_{n}\left(\chi\left(q_{0}\right) \tilde{f}\right)$ defined by the integral (6.1) is an element of $L\left(\mathscr{Q}_{4}, H\right)$, but since supp $d E \subset V_{+}$by the third axiom it really belongs to $L\left(\Theta_{\approx}\left(\bar{V}_{+}\right), H\right)$. Let $S_{0}$ be the closure of $\left\{\underline{p}_{n} ; q_{0} \in \bar{V}_{+}\right\}$in $\boldsymbol{D}^{4 n}$, then what we have just ascertained shows that $\widetilde{\Omega}_{n}\left(\underline{p}_{n}\right)$ $\in L\left(\bigotimes_{\approx}\left(S_{0}\right), H\right)$. It is readily seen that $\left\langle A^{k}, g\right\rangle \Omega_{n-k}(f)=\widetilde{\Omega}_{n}(\widetilde{g} \otimes \tilde{f})$ and

$$
\left\langle A^{k}, g\right\rangle \int \chi(p) d E(p) \widetilde{\Omega}_{n-k}(\tilde{f})=\widetilde{\Omega}_{n}\left(\widetilde{g} \otimes \chi\left(q_{k}\right) \tilde{f}\right) \quad \text { for } \quad 1 \leqq k \leqq n-1 .
$$

Let us define likewise $S_{k}$ as the closure of $\left\{p_{n} ; q_{k} \in \bar{V}_{+}\right\}$. Then $\widetilde{\Omega}_{n}\left(\underline{p}_{n}\right)$ $\in L\left(\mathcal{O}_{\mathcal{O}}\left(\cap_{\substack{n-1 \\ k=0}} S_{k}\right), H\right)$. To put this in another way we write $\widetilde{\Omega}_{n}\left(\underline{p}_{n}\right)$ $=\widetilde{\Psi}_{n}\left(q_{0}, \underline{q}_{n-1}\right)$, then we obtain $\left.\widetilde{\Psi}_{n}\left(q_{0}, \underline{q}_{n-1}\right) \in L\left(\underset{\approx}{\left(V_{+}^{n}\right.}\right), H\right)$. Since

$$
\widetilde{W}_{n}\left(\underline{p}_{n}\right)=\left(\Omega, \widetilde{\Omega}_{n}\left(\underline{p}_{n}\right)\right)=\left(\Omega, \widetilde{\Psi}_{n}\left(\underline{q}_{0}, \underline{q}_{n-1}\right)\right)=\delta\left(q_{0}\right) \widetilde{W}_{n-1}\left(\underline{q}_{n-1}\right),
$$

$\widetilde{W}_{n-1}\left(\underline{q}_{n-1}\right)$ is a Fourier hyperfunction whose support is contained in $\overline{V_{+}{ }^{n-1}}$. This completes the proof of (R5).

\subsection{Reconstruction of relativistic fields}

Theorem 6.1. To a given set of Wightman Fourier hyperfunctions satisfying $(R 0)-(R 5)$ there corresponds uniquely a neutral scalar field $A(f)$ which obeys all the axioms of Fourier hyperfunction fields and has the Wightman Fourier hyperfunctions as vacuum expectations.

Proof. Suppose $\mathscr{Q}$ be a vector space of sequence $\underline{f}=\left(f_{0}, f_{1}, \cdots\right)$, where $f_{0} \in \mathbb{C}, f_{n} \in \mathscr{Q}_{4 n}$ for $1 \leqq n \leqq N$ and $f_{n}=0$ if $n>N$ for some finite $N$. Let $(\underline{f}, \underline{g})=\sum_{n, m} \mathfrak{W}_{n+m}\left(f_{n}^{*} \times g_{m}\right)$ with $\underline{f}, \underline{g} \in \underline{\mathscr{Q}}$. Owing to (R2) this serves as a semi-definite inner product and the completion of $\mathscr{L} / \mathscr{N}$, where $\mathscr{N}=\{f \in \mathscr{L} ;(f, f)=0\}$, defines a Hilbert space $\mathcal{H}$. Let $\Phi$ be the natural 
map of $\mathscr{L}$ into $\mathcal{K}$. We set $\Omega=\Phi(1,0,0, \cdots)$. We now define a linear transformation $U(a, \Lambda)$ of $\mathscr{Q}$ by

$$
U(a, \Lambda)\left(f_{0}, f_{1}, f_{2}, \cdots\right)=\left(f_{0}, f_{1,\{a, \Lambda\}}, f_{2,\{a, \Lambda\}}, \cdots\right) .
$$

We have clearly $U(a, \Lambda) \Omega=\Omega$. A linear operator $A(f), f \in \mathcal{Q}_{4}$, is introduced by the equation

$$
A(f)\left(f_{0}, f_{1}, f_{2}, \cdots\right)=\left(0, f f_{0}, f \otimes f_{1}, f \otimes f_{2}, \cdots\right) .
$$

Then it is easily verified that $U(a, \Lambda)$ is a unitary representation of the restricted Poincare group and $A(f)$ represents the field operator having the required transformation property.

If $f$ has only one component $\underline{f}=f_{n} \in \mathscr{Q}_{4 n}$, we write formally

$$
\Phi(\underline{f}) \equiv \Phi_{n}\left(f_{n}\right)=\int \Phi_{n}\left(\underline{x}_{n}\right) f_{n}\left(\underline{x}_{n}\right) d \underline{x}_{n}
$$

$\Phi_{n}$ is a continuous linear operator from $\mathscr{Q}_{4 n}$ to $\mathcal{H}$. Upon setting $\Phi_{n}\left(\underline{x}_{n}\right)$ $=\Psi_{n}\left(x_{1}, \underline{\xi}_{n-1}\right)$ we have

$$
\left(\Psi_{n}\left(x, \underline{\xi}_{n-1}\right), \Psi_{m}\left(x^{\prime}, \underline{\xi}_{m-1}^{\prime}\right)\right)=W_{n+m-1}\left(-_{n-1} \underline{\xi},-x+x^{\prime}, \underline{\xi}_{m-1}^{\prime}\right) .
$$

Let $\widetilde{\Psi}_{n}\left(q_{0}, \underline{q}_{n-1}\right)$ be the Fourier transform of $\Psi_{n}$. Then, since $\left\|\widetilde{\Psi}_{n}(\widetilde{\phi})\right\|$ is written in terms of the Fourier hyperfunction $\widetilde{W}_{2 n-1}$, it follows from (R5) that $\widetilde{\Psi}_{n}$ is a continuous linear operator from $\left.\underset{\approx}{\mathcal{O}\left(V_{+}{ }^{n}\right.}\right)$ to $\mathcal{H}$. Thus the support of $\widetilde{\Psi}_{n}$ is contained in $\overline{V_{+}^{n}}$. For any $\chi \in \underset{\approx}{\mathcal{O}}\left(\bar{V}_{+}\right) \cap \mathscr{S}$ we have $\int_{\chi}(p) d E(p) \widetilde{\Psi}_{n}(\widetilde{\phi})=\widetilde{\Psi}_{n}\left(\chi\left(q_{0}\right) \widetilde{\phi}\right)$, where $E(p)$ is the resolution of unity associated with the energy-momentum operator $P$ and if $\operatorname{supp} \chi \cap V_{+}=\emptyset$ the right hand side of this equality vanishes. This fact shows that the spectrum of the energy-momentum operator $P$ is contained in $V_{+}$. The statement that the cluster property (R4) implies the uniqueness of the vacuum is verified in the same way as in the tempered fields [17].

Finally the locality axiom is proved as follows:

$$
\begin{gathered}
\left(A\left(x_{1}\right) A\left(x_{2}\right) A\left(u_{1}\right) \cdots A\left(u_{n}\right) \Omega-A\left(x_{2}\right) A\left(x_{1}\right) A\left(u_{1}\right) \cdots A\left(u_{n}\right) \Omega\right. \\
\left.A\left(y_{1}\right) A\left(y_{2}\right) A\left(v_{1}\right) \cdots A\left(v_{n}\right) \Omega-A\left(y_{2}\right) A\left(y_{1}\right) A\left(v_{1}\right) \cdots A\left(v_{n}\right) \Omega\right) \\
\left.=W_{2 n+4}{ }_{n} \underline{u}, x_{2}, x_{1}, y_{1}, y_{2}, \underline{v}_{n}\right)-W_{2 n+4}\left({ }_{n} \underline{u}, x_{1}, x_{2}, y_{1}, y_{2}, \underline{v}_{n}\right) \\
\quad+W_{2 n+4}\left({ }_{n} \underline{u}, x_{2}, x_{1}, y_{2}, y_{1}, \underline{v}_{n}\right)-W_{2 n+4}\left({ }_{n} \underline{u}, x_{1}, x_{2}, y_{2}, y_{1}, \underline{v}_{n}\right)=0
\end{gathered}
$$

if $\left(x_{1}-x_{2}\right)^{2}<0$ or $\left(y_{1}-y_{2}\right)^{2}<0$, i.e., if $\left(x_{1}, x_{2}, y_{1}, y_{2}\right) \in\left(K^{c} \times \mathbb{D}^{4}\right) \cup\left(\boldsymbol{D}^{4}\right.$ 
$\left.\times K^{c}\right)$, where $K$ is the closure of $\left\{\left(x_{1}, x_{2}\right) ;\left(x_{1}-x_{2}\right)^{2}>0\right\}$ in $\mathbb{D}^{8}$ and $K^{c}$ is its complement. Thus the support of the Fourier hyperfunction above is contained in $\left(K^{c} \times \mathbb{D}^{4} \cup \mathbb{D}^{4} \times K^{c}\right)^{c}=K \times K$. Therefore, if $\Phi$ is a linear combination of vectors of the form $A\left(\phi_{1}\right) \cdots A\left(\phi_{n}\right) \Omega$, then $A\left(x_{1}\right) A\left(x_{2}\right) \Phi$ $-A\left(x_{2}\right) A\left(x_{1}\right) \Phi \in L\left(\bigotimes_{\approx}(K), H\right)$, that is $A\left(x_{1}\right) A\left(x_{2}\right) \Phi=A\left(x_{2}\right) A\left(x_{1}\right) \Phi$ if $x_{1}$ and $x_{2}$ are spacelike separated.

\section{§ 7. Euclidean Green's Functions for Fourier Hyperfunction Fields}

The analytic continuation of Wightman functions into Euclidean points is carried out rather straightforwards and we can derive its properties, which we shall again state in the form of the axioms for Euclidean Green's functions. In $\S 7$ and 8 , we denote by $V_{+}$the forward light cone.

Proposition 7.1. If $\mu \in \underset{\approx}{\mathcal{Q}}\left(\overline{V_{+}^{n}}\right)$, then $F\left(\zeta_{n}\right)=\mu\left(\exp \left\{i\left((\cdot, \zeta)_{n}\right\}\right)\right.$ is holomorphic in the tube $\mathfrak{I}_{+}^{n} \equiv \boldsymbol{R}^{4 n} \times i{V_{+}}^{n}=\left\{\zeta_{n} \in \mathbb{C}^{4 n} ; \operatorname{Im} \zeta_{n} \in V_{+}{ }^{n}\right\}$, and in the Euclidean points $\left\{\zeta_{n} \in \mathbb{C}^{4 n} ; \operatorname{Re} \zeta_{n}{ }^{0}=0, \operatorname{Im} \zeta_{n}=0\right\}$ it satisfies the condition that

$$
\left|F\left(\iota \underline{\zeta}_{n}\right)\right| \leqq C_{\varepsilon} e^{\varepsilon|\underline{\xi}|}
$$

is valid for every $\varepsilon>0$ and $\xi_{n} \in Q_{4 \varepsilon}^{n}$, where $Q_{4 \varepsilon}=\left\{\xi \in \mathbb{R}^{4} ; \xi^{0}>4 \varepsilon(1+|\xi|)\right\}$ and $\xi_{n}=\operatorname{Re} \zeta_{n}$.

Proof. It is readily seen that $\exp \left\{i\left((\cdot, \zeta)_{n}\right\}\right.$ for $\zeta_{n} \in \mathfrak{I}_{+}{ }^{n}$ belongs to $\underset{\approx}{\mathcal{O}}\left(\overline{V_{+}{ }^{n}}\right)$. Hence $\mu\left(\exp \left\{i\left((\cdot, \zeta)_{n}\right\}\right)\right.$ is defined well and holomorphic there. Let $z=x+i y$ and consider

$$
\Delta_{\varepsilon}=\left\{x^{0}+\varepsilon>(1-\varepsilon)(x \cdot x)^{1 / 2},\left|y^{\mu}\right|<\varepsilon\left(1+\left|x^{\mu}\right|\right) \text { for } 0 \leqq \mu \leqq 3\right\},
$$

then $\Delta_{\varepsilon}^{n}$ is a neighbourhood of $V_{+}^{n}$. If $\zeta_{n} \in Q_{4 \varepsilon}^{n}$ we have (see $\S 2$ for notations)

$$
\begin{aligned}
\left|F\left(\iota \xi_{n}\right)\right| & \leqq C_{\varepsilon}{ }^{\prime} \sup _{z \in \Delta_{\varepsilon}^{n}}\left|e^{i\left((z,(\xi))_{n}+\varepsilon\left|\underline{z}_{n}\right|\right.}\right| \\
& \leqq C_{\varepsilon}{ }^{\prime} \sup _{z \in \Delta_{\varepsilon}^{n}} \exp \left\{-\left(\langle y, \xi)_{n}-\left(\left\langle x^{0}, \xi^{0}\right)\right)_{n}+\varepsilon\left|\underline{x}_{n}\right|+\varepsilon\left|y_{n}\right|\right\} .\right.
\end{aligned}
$$

We now put $x^{\prime}=\left(x^{0}+\varepsilon, x\right)$, then we have $x^{\prime 0}>(1-\varepsilon)\left(x^{\prime} \cdot x^{\prime}\right)^{1 / 2}$. 
Further it is possible to put $y^{\prime}=y-\alpha$ such that $\left|y^{\prime \mu}\right|<\varepsilon\left|x^{\mu}\right|$ and $\left|\alpha^{\mu}\right|<\varepsilon$. Let $V_{\varepsilon}=\left\{s \equiv\left(x^{\prime 0}, y^{\prime}\right) ;\left(y^{\prime} \cdot y^{\prime}\right)<(\varepsilon / 1-\varepsilon) x^{\prime 0}\right\}$ and $W_{\varepsilon}=\left\{\xi^{\prime} ; \xi^{\prime 0}>4 \varepsilon\left|\xi^{\prime}\right|\right\}$, the $\left(\left(s, \xi^{\prime}\right)\right\rangle_{n} \geqq 0$ for $\underline{s}_{n} \in V_{\varepsilon}^{n}, \quad \underline{\xi}_{n}{ }^{\prime} \in W_{\varepsilon}^{n}$ and $0<\varepsilon<1 / 8$. Coming back to the estimate we obtain

$$
\begin{aligned}
& \leqq C_{\varepsilon}^{\prime \prime} \sup _{\underline{s}_{n} \in V_{\varepsilon}{ }^{n},\left|\alpha^{\prime \prime}\right|<\varepsilon} \exp \left\{-\left(\left(\left(x^{\prime 0}-\varepsilon, y^{\prime}+\alpha\right),\left(\xi^{\prime 0}+4 \varepsilon, \xi^{\prime}\right)\right)_{n}\right.\right. \\
& \left.\quad+\varepsilon\left|\underline{x}_{n}\right|+\varepsilon\left|\underline{y}_{n}^{\prime}+\underline{\alpha}_{n}\right|\right\} \\
& \leqq C_{\varepsilon}{ }^{\prime \prime} \sup _{\underline{s}_{n} \in V_{\varepsilon^{n}}} \exp \left\{-\left(\left(s, \xi^{\prime}\right)\right)_{n}+\varepsilon\left|\underline{\xi}_{n}\right|-4 \varepsilon\left|\underline{x}_{n}{ }^{\prime 0}\right|+3 \varepsilon(1+\varepsilon)\left|\underline{x}_{n}{ }^{\prime 0}\right|\right\} \\
& \leqq C_{\varepsilon} e^{\varepsilon\left|\underline{\xi}_{n}\right|},
\end{aligned}
$$

which completes the proof.

By the relativistic covariance and the Bargmann-Hall-Wightman theorem we obtain a single-valued analytic extension of the Wightman function $W_{n-1}\left(\zeta_{n-1}\right)$ into the extended tube $\mathfrak{I}_{+, \text {ext }}^{n-1}=\left\{\underline{\zeta}_{n-1} \in C^{4(n-1)} ; \Lambda \underline{\zeta}_{n-1}\right.$ $\in \mathfrak{T}_{+}^{n-1}$ for some $\left.\Lambda \in L_{+}(\boldsymbol{C})\right\}$, where $L_{+}(\boldsymbol{C})$ is the set of all complex proper Lorentz transformations and $\zeta_{j}=z_{j+1}-z_{j}, 1 \leqq j \leqq n-1$. The function $\mathfrak{M}_{n}\left(z_{n}\right)$, defined by $\mathfrak{M}_{n}\left(z_{n}\right)=W_{n-1}\left(\zeta_{n-1}\right)$, is analytic in $\sigma_{\text {ext }}^{n}=\left\{z_{n} \in \mathbb{C}^{4 n}\right.$; $\left.\zeta_{n-1} \in \mathfrak{I}_{+, \text {ext }}^{n-1}\right\}$ and has the Fourier hyperfunction $\mathfrak{I}_{n}\left(\underline{x}_{n}\right)$ as its boundary value. Using the locality (R3) we obtain a single-valued analytic extension of $\mathfrak{M}_{n}\left(z_{n}\right)$ into the set $\sigma_{\text {ext, perm }}^{n}=\left\{z_{n} \in \mathbb{C}^{4 n} ; z_{\pi(n)} \in \sigma_{\text {ext }}^{n}\right.$ for some permutation $\pi\}$, where $z_{\pi(n)}=\left(z_{\pi(1)}, \cdots, z_{\pi(n)}\right)$ and $(\pi(1), \cdots, \pi(n))$ is a permutation of $(1, \cdots, n)$. We denote this extension again by $\mathfrak{M}_{n}\left(z_{n}\right)$. It is invariant under the complex Poincaré group and also under permutations of the arguments $z_{1}, \cdots, z_{n}$. The set $\sigma_{\text {ext,perm }}^{n}$ contains the Set of Euclidean points (of noncoinciding arguments) $E^{n}=\left\{\underline{z}_{n} \in \mathbb{C}^{4 n} ; \operatorname{Re} \underline{z}_{n}{ }^{0}=0\right.$, $\operatorname{Im} \underline{z}_{n}=0$ and $z_{i} \neq z_{j}$ if $\left.i \neq j\right\}$.

Definition 7.2. The restriction of the Wightman function $\mathfrak{M}_{n}\left(z_{n}\right)$ to $E^{n}$ is called the $n$-point Euclidean Green's function or the Schwinger function.

We set $\mathfrak{S}_{0}=\mathfrak{M}_{0}=1$ and

$$
\mathfrak{S}_{n}\left(\underline{x}_{n}\right)=\mathfrak{W}_{n}\left(\iota \underline{x}_{n}\right),
$$




$$
S_{n-1}\left(\underline{\xi}_{n-1}\right)=W_{n-1}\left(\iota \underline{\xi}_{n-1}\right)=\Im_{n}\left(\underline{x}_{n}\right),
$$

where $\underline{x}_{n} \in \boldsymbol{R}^{4 n}, x_{i} \neq x_{j}$ if $i \neq j$. Then we can derive the following properties of Schwinger functions.

\section{Distribution property.}

$$
\begin{aligned}
\mathfrak{S}_{0}=1, \mathfrak{S}_{n}\left(\underline{x}_{n}\right) \in\left(\mathscr{C}_{0}\left(\boldsymbol{R}^{4 n}\right)\right)^{\prime} \text { and } \\
\\
\left.\quad \overline{\mathfrak{S}_{n}\left(\underline{x}_{n}\right)}=\mathfrak{S}_{n}\left(\theta_{n} \underline{x}\right)\right) \text { for each } n \geqq 1 .
\end{aligned}
$$

Proof. Since by Proposition 7.1

$$
\sup _{\underline{\xi}_{n-1} \in Q_{4} \varepsilon^{n-1}}\left|S_{n-1}\left(\underline{\xi}_{n-1}\right)\right| e^{-\varepsilon\left|\underline{\xi}_{n-1}\right|}<\infty \quad \text { for all } \varepsilon>0,
$$

it is evident that $S_{n-1} \in\left(\mathscr{C}_{+}\left(\boldsymbol{R}^{4(n-1}\right)^{\prime}\right.$. With the aid of a geometrical argument of Osterwalder and Schrader [13] we have the distribution property. The last statement of (E0) follows immediately from $\overline{\mathfrak{W}_{n}\left(\underline{x}_{n}\right)}$ $=\mathfrak{W}_{n}\left({ }_{n} \underline{x}\right)$.

\section{Euclidean convariance.}

$$
\mathfrak{S}_{n}\left(\underline{x}_{n}\right)=\mathfrak{S}_{n}\left(R \underline{x}_{n}+a\right)
$$

for each $n \geq 1$ and all $(a, R) \in i S O_{4}$, the inhomogeneous Euclidean group.

\section{Positivity.}

$$
\sum_{n, m} S_{n+m}\left(\Theta f_{n}^{*} \times f_{m}\right) \geqq 0
$$

for all finite sequences $f_{0}, f_{1}, \cdots, f_{N}$ of test functions $f_{0} \in \boldsymbol{C}$ and $f_{n} \in \mathscr{C}_{<}\left(\boldsymbol{R}^{4 n}\right)$, $n \geqq 1$. Here $\Theta f_{n}\left(\underline{x}_{n}\right)=f_{n}\left(\theta \underline{x}_{n}\right)$.

\section{Symmetry.}

$$
\mathfrak{S}_{n}\left(\underline{x}_{n}\right)=\mathfrak{S}_{n}\left(\underline{x}_{\pi(n)}\right)
$$

for all permutations $\pi:(1, \cdots, n) \rightarrow(\pi(1), \cdots, \pi(n))$.

Cluster porperty.

$$
\lim _{\lambda \rightarrow \infty} \mathfrak{S}_{n+m}\left(f \times g_{\lambda a}\right)=\mathfrak{S}_{n}(f) \mathfrak{S}_{m}(g),
$$


where $f \in \mathscr{C}_{<}\left(\boldsymbol{R}^{4 n}\right), g \in \mathscr{C}_{<}\left(\boldsymbol{R}^{4 m}\right)$ and $a=(0, \boldsymbol{a})$.

For the proof of these four properties of Schwinger functions we only refer to NM II.

Proposition 7.3. The correspondence from Wightman functions (Fourier hyperfunctions) to Schwinger functions (distributions) is one to one.

Proof. Suppose that

$$
\int W_{n-1}\left(\iota \underline{\xi}_{n-1}\right) g\left(\underline{\xi}_{n-1}\right) d \underline{\xi}_{n-1}=0 \text { for all } g \in \mathscr{C}_{+}\left(\boldsymbol{R}^{4(n-1)}\right),
$$

then $W_{n-1}\left(\iota \underline{\xi}_{n-1}\right)=0$ for $\underline{\xi}_{n-1}^{0}>0$. Since $W_{n-1}\left(\iota \underline{\xi}_{n-1}\right)$ is a real analytic function, $W_{n-1}\left(\underline{\zeta}_{n-1}\right)=0$ if $\operatorname{Im} \underline{\zeta}_{n-1} \in V_{+}{ }^{n-1}$. By the uniqueness of Fourier transformation of Fourier hyperfunctions we have $\widetilde{W}_{n-1}=0$.

\section{§ 8. Equivalence of Relativistic and Euclidean Theories}

In the preceding section, from a given set of Wightman Fourier hyperfunctions satisfying the axioms (R0)-(R5), we have constructed a set of Schwinger functions possessing the properties (E0)-(E4). Conversely we can prove the following theorem.

Theorem 8.1. (Reconstruction of the relativistic theory). To a given sequence of Euclidean Green's functions satisfying (EO)-(E4) there corresponds uniquely a sequence of Wightman Fourier hyperfunctions having the properties (RO)-(R5) and whose Schwinger functions coincide with the Euclidean Green's functions given initially.

Remark. In NM II we have proved the same theorem for the case of a smaller class in which Wightman Fourier hyperfunctions are of the first type for spatial variables, while they are of the second type for temporal variables.

As in $\S 6.2$ we begin with constructing a Hilbert space. Let $\underline{\mathscr{C}}<$ be 
a vector space of sequences $\underline{f}=\left(f_{0}, f_{1}, \cdots\right)$, where $f_{0} \in \boldsymbol{C}, f_{n} \in \mathscr{C}_{<}\left(\boldsymbol{R}^{4 n}\right)$ for $1 \leqq n \leqq N$ and $f_{n}=0$ if $\left.n\right\rangle N$ for some finite $N$. Let $\langle\underline{f}, \underline{q}\rangle=\sum_{n, m} \Im_{n+m}$ $\left(\theta f_{n}{ }^{*} \times g_{m}\right)$ with $\underline{f}, \underline{g} \in \underline{\mathscr{C}}_{<}$. Owing to (E2) this serves as a semi-definite inner product and the completion of $\underline{\mathscr{C}}_{<} / \mathscr{I}$, where $\Re=\{\underline{f} \in \underline{\mathscr{C}}<;\langle\underline{f}, \underline{f}\rangle$ $=0\}$, defines a Hilbert space $\mathcal{K}$. Let $\Phi^{E}$ be the natural map of $\underline{\mathscr{C}}_{<}$ into $\mathcal{K}$. We obtain $\left(\Phi^{E}(\underline{f}), \Phi^{E}(\underline{g})\right)=\langle\underline{f}, \underline{q}\rangle$. We set $\Omega=\Phi^{E}(1,0,0, \cdots)$. For $\underline{f} \in \underline{\mathscr{C}}_{<}$and $a=(0, \boldsymbol{a}) \in \boldsymbol{R}^{4}$ we define $\widehat{U}_{s}(a) \underline{f}$ by $\left(\widehat{U}_{s}(a) \underline{f}\right)_{n}\left(\underline{x}_{n}\right)$ $=f_{n}\left(\underline{x}_{n}-a\right)$. We can extend it to a unitary operator $U_{s}(a)$ in $\mathcal{K}$ by (E1) (see Osterwalder-Schrader [13]). If $\underline{f}$ has only one nonvanishing component $\underline{f} \equiv f_{n} \in \mathscr{C}_{<}\left(\boldsymbol{R}^{4 n}\right)$, we write formally

$$
\Phi^{E}(\underline{f}) \equiv \Phi_{n}{ }^{E}\left(f_{n}\right)=\int \Phi_{n}{ }^{E}\left(\underline{x}_{n}\right) f_{n}\left(\underline{x}_{n}\right) d \underline{x}_{n}
$$

Let us define $\Psi_{n}{ }^{E}\left(x_{1}, \underline{\xi}_{n-1}\right)=\Phi_{n}{ }^{E}\left(\underline{x}_{n}\right)$, then it is a vector-valued distribution over $\mathscr{C}_{+}\left(\boldsymbol{R}^{4 n}\right)$ and we have by (E2)

$$
\begin{aligned}
& \left(\Psi_{n}^{E}\left(x_{1}, \underline{\xi}_{n-1}\right), \Psi_{m}^{E}\left(x_{1}^{\prime}, \underline{\xi}_{m-1}^{\prime}\right)\right) \\
& \quad=S_{n+m-1}\left(-\theta\left(_{n-1} \underline{\xi}\right),-\theta x_{1}+x_{1}^{\prime}, \underline{\xi}_{m-1}^{\prime}\right)=\Im_{n+m}\left(\theta\left(_{n} \underline{x}\right), \underline{x}_{m}{ }^{\prime}\right) .
\end{aligned}
$$

Lemma 8.2. For $t \geqq 0$ we define $\widehat{T}_{t}: \underline{\mathscr{C}}_{<} \rightarrow \underline{\mathscr{C}}_{<}$by $\left(\widehat{T}_{t} \underline{f}\right)_{n}\left(\underline{x}_{n}\right)=$ $\underline{f}_{n}\left(\underline{x}_{n}-t\right)$, where $t=(t, \mathbf{0})$. Then $\widehat{T}_{t}$ induces a continuous one-parameter semigroup of self-adjcint contraction operators $\left\{T_{t}\right\}$ on $\mathcal{K}$.

\section{Proof. See NM II.}

Let $-H$ be the infinitesimal generator of $T_{t}$, then

$$
e^{-t H} \Psi_{n}^{E}\left(x, \xi_{n-1}\right)=\Psi_{n}^{E}\left(x+t, \xi_{n-1}\right) \quad \text { for } \quad t \geqq 0 .
$$

Furthermore for $\tau \in \boldsymbol{C}_{+} \equiv \boldsymbol{R}_{+}+i \boldsymbol{R}$

$$
\begin{aligned}
& \left\langle\Psi_{n}^{E}\left(x, \underline{\underline{\xi}}_{n-1}\right), e^{-\tau H} \Psi_{m}^{E}\left(x^{\prime}, \underline{\underline{\xi}}_{m-1}^{\prime}\right)\right\rangle \\
& \quad=S_{n+m-1}\left(-\theta\left(_{n-1} \underline{\xi}\right),\left(x^{0}+x^{\prime 0}+\tau,-\boldsymbol{x}+\boldsymbol{x}^{\prime}\right), \underline{\xi}_{m-1}^{\prime}\right)
\end{aligned}
$$

defines an analytic continuation of $S_{n+m-1}$ in the n'th time-variable.

Before entering into the analytic continuation of Euclidean Green's functions we provide some definitions of domains. For $N=0,1,2, \cdots$ we define 


$$
\begin{aligned}
\Gamma_{k, \varepsilon}^{(N)}= & \left\{\xi_{k}+\underline{\omega}_{k} ; \xi_{k} \in \mathbb{R}^{4 k}, \underline{\omega}_{k} \in \mathbb{C}^{4 k}, \xi_{i}{ }^{0}>\varepsilon\left|\xi_{i}\right|,\right. \\
& \left.\left|\omega_{i}{ }^{\mu}\right|<\pi \varepsilon \xi_{i}{ }^{0} / 2^{9} \cdot 2^{N} \cdot k \text { for } \quad 1 \leqq i \leqq k, 0 \leqq \mu \leqq 3\right\} .
\end{aligned}
$$

Next two sets of domains $C_{k}{ }^{(N)}$ and $D_{n}{ }^{(N)}, N=0,1,2, \cdots$ are defined successively by commencing with

$$
C_{k}{ }^{(0)}=\left\{\rho_{k}{ }^{0} \in \mathbb{R}^{k} ; \arg \rho_{k}{ }^{0}=0\right\} ;
$$

then follows

$$
D_{n}{ }^{(N)}=\left\{\left(x^{0}, \underline{\rho}_{n-1}^{0}\right) ; x^{0}>0,\left({ }_{n-1} \underline{\rho}^{0}, 2 x^{0}, \underline{\rho}_{n-1}^{0}\right) \in C_{2 n-1}^{(N)}\right\} .
$$

Therefore we have $D_{n}{ }^{(0)}=\left\{\left(x^{0}, \underline{\rho}_{n-1}^{0}\right) ; x^{0}>0\right.$, arg $\left.\underline{\rho}_{n-1}^{0}=0\right\} . C_{k}{ }^{(N)}$ is the envelope of holomorphy of $\widehat{C}_{k}^{(N)}$ :

$$
\begin{array}{r}
\widehat{C}_{k}^{(N)}=\bigcup_{j=1}^{k}\left\{\left({ }_{j-1} \underline{\rho}^{0}, x^{0}+x^{\prime 0}+z, \underline{\rho}_{k-j}^{\prime 0}\right) ; z \in C_{+},\right. \\
\left.\left(x^{0}, \underline{\rho}_{j-1}^{0}\right) \in D_{j}^{(N-1)},\left(x^{\prime}{ }_{0}, \underline{\rho}_{k-j}^{\prime 0}\right) \in D_{k-j+1}^{(N-1)}\right\} .
\end{array}
$$

Lemma 8.3. The distribution $S_{k}\left(\xi_{k}\right)$ is the restriction to $\left(\boldsymbol{R}_{+}\right.$ $\left.\times \boldsymbol{R}^{3}\right)^{k}$ of a function $S_{k}\left(\underline{\zeta}_{k}\right)$ analytic in $\underline{\zeta}_{k}=\underline{\xi}_{k}+\underline{\omega}_{k} \in \Gamma_{k, \varepsilon}^{(0)}$

Proof. This follows from the inequality $(5 \cdot 14)$ in the proof of Lemma 5.1 of Osterwalder and Schrader [14].

Lemma 8.4. The following statements $\left(\mathrm{A}_{N}\right)$ and $\left(\mathrm{P}_{N}\right)$ are true for $N=0,1,2, \cdots$.

$\left(\mathrm{A}_{N}\right)$ There is an analytic continuation $S_{k}\left(\underline{\zeta}_{k}\right)$ of $S_{k}\left(\underline{\xi}_{k}\right)$, analytic in $\underline{\zeta}_{k} \in \Gamma_{k, \varepsilon}^{(N)}+C_{k}{ }^{(N)}$. Here $\underline{\zeta}_{k} \in \Gamma_{k, \varepsilon}^{(N)}+C_{k}{ }^{(N)}$ means that on writing $\underline{\zeta}_{k}=\underline{\xi}_{k}$ $+\underline{\omega}_{k}+\underline{\rho}_{k}$, we have $\underline{\xi}_{k}+\underline{\omega}_{k} \in \Gamma_{k, \varepsilon}^{(N)}$ and $\underline{\rho}_{k}=\left(\underline{\rho}_{k}{ }^{0}, \underline{\mathbf{\theta}}_{k}\right), \underline{\rho}_{k}{ }^{0} \in C_{k}{ }^{(N)}$.

$\left(\mathrm{P}_{N}\right)$ There are $H$-valued functions $\Psi_{n}{ }^{E}\left(z, \underline{\zeta}_{n-1}\right)$ defined for ( $z$, $\left.\zeta_{n-1}\right) \in \Gamma_{n, \varepsilon}^{(N+1)}+D_{n}{ }^{(N)}$ such that the scalar product is given by

$$
\left\langle\Psi_{n}^{E}\left(z, \underline{\zeta}_{n-1}\right), \Psi_{m}^{E}\left(z^{\prime}, \underline{\zeta}_{m-1}^{\prime}\right)\right\rangle=S_{n+m-1}\left(-\theta\left(_{n-1} \underline{\bar{\zeta}}\right),-\theta \bar{z}+z^{\prime}, \underline{\zeta}_{m-1}^{\prime}\right) .
$$

Here $\left(z, \zeta_{n-1}\right) \in \Gamma_{n, \varepsilon}^{(N+1)}+D_{n}{ }^{(N)}$ means that on writing $z=\xi_{n}+\omega_{n}+\left(x^{0}, \mathbf{0}\right)$, $\underline{\zeta}_{n-1}=\underline{\xi}_{n-1}+\underline{\omega}_{n-1}+\underline{\rho}_{n-1}$, we have $\underline{\xi}_{n}+\underline{\omega}_{n} \in \Gamma_{n, \varepsilon}^{(N+1)}$ and $\left(x^{0}, \underline{\rho}_{n-1}^{0}\right) \in D_{n}{ }^{(N)}$.

Proof. $\left(\mathrm{P}_{N}\right)$ follows from $\left(\mathrm{A}_{N}\right)$ in the same way as OsterwalderSchrader [14]. Hence it suffices to establish $\left(A_{N}\right)$. We prove $\left(A_{N}\right)$ by induction. $\left(\mathrm{A}_{0}\right)$ is nothing but the statement of Lemma 8.3. Now 
assuming that $\left(\mathrm{A}_{N}\right)$ and $\left(\mathrm{P}_{N}\right)$ have been verified for $0 \leqq N \leqq M-1$, we will prove $\left(\mathrm{A}_{M}\right)$. By $\left(\mathrm{P}_{M-1}\right)$ we can define

$$
\begin{aligned}
S_{n+m-1}( & \left.-\theta\left(_{n-1} \bar{\zeta}\right),-\theta \bar{z}+z^{\prime}+\tau, \underline{\zeta}_{m-1}^{\prime}\right) \\
& =\left\langle\Psi_{n}^{E}\left(z, \underline{\zeta}_{n-1}\right), e^{-\tau H} \Psi_{m}^{E}\left(z^{\prime}, \underline{\zeta}_{m-1}^{\prime}\right)\right\rangle
\end{aligned}
$$

for $\left(z, \underline{\zeta}_{n-1}\right) \in \Gamma_{n, \varepsilon}^{(M)}+D_{n}^{(M-1)}$ and $\left(z^{\prime}, \underline{\zeta}_{m-1}^{\prime}\right) \in \Gamma_{m}^{(M)}+D_{m}^{(M-1)}$. This equation makes it possible to extend $S_{k}, k=n+m+1$, analytically to $\Gamma_{k, \varepsilon}^{(M)}+C_{k}{ }^{(M)}$. In fact, let $\zeta_{k}=\zeta_{k}{ }^{\prime}+\zeta_{k}{ }^{\prime \prime}$ such that $\zeta_{k}{ }^{\prime} \in \Gamma_{k, \varepsilon}^{(M)}$ and $\zeta_{k}{ }^{\prime \prime} \in \widehat{C}_{k}{ }^{(M)}$, then $S_{k}\left(\zeta_{k}{ }^{\prime}\right.$ $\left.+\zeta_{k}{ }^{\prime \prime}\right)$ for fixed $\zeta_{k}{ }^{\prime}$ is analytic in $\zeta_{k}{ }^{\prime \prime} \in \widehat{C}_{n}{ }^{(M)}$ and hence, because $C_{k}{ }^{(M)}$ is the envelope of holomorphy of $\widehat{C}_{k}{ }^{(M)}$, there is an analytic extension of $S_{k}\left(\zeta_{k}{ }^{\prime}+\zeta_{k}{ }^{\prime \prime}\right)$ into $\zeta_{k}{ }^{\prime \prime} \in C_{k}{ }^{(M)}$, and this extension depends analytically on $\zeta_{k}{ }^{\prime} \in \Gamma_{k, \varepsilon}^{(M)}$. Thus $S_{k}\left(\zeta_{k}\right)$ has been proved to be analytic in $\zeta_{k} \in \Gamma_{k, \varepsilon}^{(M)}+C_{k}{ }^{(M)}$ which establishes $\left(A_{M}\right)$ as required.

Lemma 8.5 (Real estimate). The function $S_{k}\left(\xi_{k}\right)$ satisfies the inequality

$$
\left|S_{k}\left(\underline{\xi}_{k}\right)\right| \leqq A_{k}, \varepsilon e^{\varepsilon\left|\underline{\xi}_{k}\right|} \text { for } \underline{\xi}_{k} \in Q_{\varepsilon}{ }^{k} \text { and any } \varepsilon>0,
$$

where $Q_{\varepsilon}$ is the cone given in Proposition 7.1, replacing $4 \varepsilon$ by $\varepsilon$.

The proof of this lemma is carried out in a way similar to that for Theorem 4.1 of Osterwalder and Schrader [14].

Lemma 8.6 (Infra-exponential estimate). For a fixed $\zeta_{k}-\varepsilon$ $\equiv\left(\underline{\zeta}_{k}{ }^{0}-\varepsilon, \underline{\zeta}_{k}\right) \in \Gamma_{k, \varepsilon}^{(N)}$ we define

$$
S_{k, \varepsilon}\left(\underline{\rho}_{k}^{0} \mid \underline{\zeta}_{k}\right)=\prod_{i=1}^{k} e^{-\varepsilon \rho_{i}{ }^{0}} S_{k}\left(\underline{\zeta}_{k}{ }^{0}+\underline{\rho}_{k}^{0}, \underline{\zeta}_{k}\right),
$$

then there is a constant $A_{k, \varepsilon}^{(N)}$ independent of $\zeta_{k}$ and $\rho_{k}{ }^{0}$ such that the following inequality holds for $\underline{\rho}_{k}{ }^{0} \in C_{k}{ }^{(N)}$ :

$$
\left|S_{k, \varepsilon}\left(\underline{\rho}_{k}^{0} \mid \underline{\zeta}_{k}\right)\right| \leqq A_{k, \varepsilon}^{(N)} e^{\varepsilon|\underline{\zeta}|_{k}} .
$$

Proof. We shall prove the lemma by induction. In the case $N=0$ case we let $\underline{\xi}_{k}$ be fixed so that each $\xi_{i}$ is in the cone $Q_{\varepsilon}$ and let $e_{\mu}$, $\mu=0,1,2,3$, be four linearly independent vectors in the dual cone $\Gamma^{\circ}=\left\{x^{0}\right.$ $>|x| / \varepsilon\}$, then by $(8 \cdot 11)$ and the Euclidean invariance of $S_{k}$ the inequality 


$$
\left|S_{k}\left(\underline{\xi}_{k}+\underline{w}_{k} \circ e\right) e^{-\varepsilon \underline{w}_{k} \circ e}\right| \leqq A_{k, \varepsilon} e^{\varepsilon\left|\underline{\xi}_{k}\right|}
$$

holds for $\underline{w}_{k} \in \cup_{i, \mu} T_{k ; i, \mu}$, where $\underline{w}_{k} \circ e$ is the set of vectors with components $\left(w_{i} \circ e\right)^{\mu}=\sum_{\nu=0}^{3} w_{i}^{\nu}\left(e_{\nu}\right)^{\mu}$ and

$$
T_{k ; i, \mu}=\left\{w_{k} ;\left|\arg w_{i}^{\mu}\right|<\pi / 2, \arg w_{\jmath}{ }^{\nu}=0 \text { for } \nu \neq \mu \text { or } j \neq \mathrm{i}\right\} .
$$

By the maximum principle the inequality (8.14) holds also for $w_{k} \in T_{k}$ $=\left\{w_{k} ; \sum_{i, \mu}\left|\arg w_{i}^{\mu}\right|<\pi / 2\right\}$. Therefore we have

$$
\left|S_{k}\left(\zeta_{k}\right)\right| \leqq A_{k, \varepsilon} e^{\varepsilon\left|\underline{\zeta}_{k}\right|} \quad \text { for } \quad \zeta_{k}-\varepsilon \in \Gamma_{k, \varepsilon}^{(0)},
$$

since $\Gamma_{k, \varepsilon}^{(0)} \subset T_{k}$ (see Lemma 5.1 of Osterwalder and Schrader [14]). Thus the lemma has been proved for $N=0$.

Assuming that the inequality $(8 \cdot 13)$ has been verified for $0 \leqq N$ $\leqq M-1$, we will prove it for $M$. For $\left(z-\varepsilon / 2, \zeta_{n-1}-\varepsilon\right) \in \Gamma_{n, \varepsilon}^{(M)},\left(z^{\prime}-\varepsilon / 2\right.$, $\left.\zeta_{m-1}^{\prime}-\varepsilon\right) \in \Gamma_{m, \varepsilon}^{(M)}$ we have

$$
\Psi_{n, \varepsilon}^{E}\left(x^{0}, \rho_{n-1}^{0} \mid z, \zeta_{n-1}\right)=e^{-\varepsilon x^{0}} \prod_{i=1}^{n-1} e^{-\varepsilon \rho 0_{i}} \Psi_{n}^{E}\left(z^{0}+x^{0}, z, \zeta_{n-1}^{0}+\rho_{n-1}^{0}, \zeta_{n-1}\right)
$$

and similarly $\Psi_{m, \varepsilon}^{E}\left(x^{\prime 0}, \rho_{n-1}^{\prime 0} \mid z^{\prime}, \zeta_{m-1}^{\prime}\right)$. Since $\left(-\theta\left(_{n-1} \bar{\zeta}-\varepsilon\right),-\theta \bar{z}+z-\varepsilon\right.$, $\left.\underline{\zeta}_{n-1}-\varepsilon\right) \in \Gamma_{2 n-1, \varepsilon}^{(M-1)}$ and $\left(-\theta\left(_{m-1} \bar{\zeta}^{\prime}-\varepsilon\right),-\theta \bar{z}^{\prime}+z^{\prime}-\varepsilon, \underline{\zeta}_{m-1}^{\prime}-\varepsilon\right) \in \Gamma_{2 m-1, \varepsilon}^{(M-1)}$, we have

$$
\begin{aligned}
&\left|S_{n+m-1, \varepsilon}\left({ }_{n-1} \underline{\rho}^{0}, x^{0}+x^{\prime 0}+i t, \underline{\rho}_{m-1}^{\prime 0} \mid-\theta\left(_{n-1} \bar{\zeta}\right),-\theta \bar{z}+z^{\prime}, \underline{\zeta}_{m-1}^{\prime}\right)\right| \\
&=\left|\left\langle\Psi_{n, \varepsilon}^{E}\left(x^{0}, \rho_{n-1}^{0} \mid z, \zeta_{n-1}\right), e^{-i t H} \Psi_{m, \varepsilon}^{E}\left(x^{\prime 0}, \rho_{m-1}^{\prime 0} \mid z^{\prime}, \zeta_{m-1}^{\prime}\right)\right\rangle\right| \\
& \leqq S_{2 n-1, \varepsilon}\left({ }_{n-1} \rho^{0}, 2 x^{0}, \rho_{n-1}^{0} \mid-\theta\left(_{n-1} \bar{\zeta}\right),-\theta \bar{z}+z, \zeta_{n-1}\right)^{1 / 2} \\
&\left.\times S_{2 m-1, \varepsilon}{ }_{m-1} \rho^{\prime 0}, 2 x^{\prime 0}, \rho_{m-1}^{\prime 0} \mid-\theta\left(_{m-1} \bar{\zeta}\right),-\theta \bar{z}^{\prime}+z^{\prime}, \zeta_{m-1}^{\prime}\right)^{1 / 2} \\
& \leqq\left(A_{2 n-1, \varepsilon}^{(M-1)} \cdot A_{2 m-1, \varepsilon}^{(M-1)}\right)^{1 / 2} \exp \left\{\left|\underline{\zeta}_{n-1}\right|+|z|+\left|\underline{\zeta}_{m-1}^{\prime}\right|+\left|z^{\prime}\right|\right\}
\end{aligned}
$$

for $\left(x^{0}, \rho_{n-1}^{0}\right) \in D_{n}{ }^{(M-1)}$ and $\left(x^{\prime 0}, \rho_{m-1}^{\prime 0}\right) \in D_{m}{ }^{(M-1)}$. If we define $A_{k, \varepsilon}^{(M)}$ by

$$
A_{k, \varepsilon}^{(M)}=\max _{n+m-1=k}\left\{\left(A_{2 n-1, \varepsilon}^{(M-1)} \cdot A_{2 m-1, \varepsilon}^{(M-1)}\right)^{1 / 2}\right\}
$$

we have the desired estimate, because $C_{k}{ }^{(M)}$ is the envelope of holomorphy of $\widehat{C}_{k}{ }^{(M)}$. Thus we have completed the proof of the lemma.

Now, define $W_{k}\left(\zeta_{k}\right)=S_{k}\left(\theta\left(\iota \zeta_{k}\right)\right)$, then $W_{k}\left(\zeta_{k}\right)$ is analytic in $\iota\left(\Gamma_{k, \varepsilon}^{(N)}\right.$ $+C_{k}{ }^{(N)}$ for any $N$. We shall show that $W_{k}\left(\zeta_{k}\right)$ determines a Fourier hyperfunction. 
Lemma 8.7. For any positive $\varepsilon$, there exists a linear transformation $L_{\varepsilon}{ }^{(N)}$ which maps the domain $\left\{\operatorname{Im} w^{\mu}>\varepsilon\left|\operatorname{Re} w^{\mu}\right|, \mu=0, \cdots, 3\right\}$ into $c\left(\Gamma_{k, \varepsilon}^{(N)}+C_{1}^{(N)}\right)$.

Proof. From Lemma 5.2 of Osterwalder and Schrader [14] it follows that for any $\varepsilon>0$ there exists $C_{1}{ }^{(N)}$ which contains $\{z \in \mathbb{C}$; $\operatorname{Re} z$ $>(\varepsilon / 2)|\operatorname{Im} z|\}$. Let us define a linear transformation by

$$
\begin{aligned}
L_{\varepsilon}{ }^{(N)} w & =\left(\begin{array}{rrrr}
\beta & \beta & \beta & \beta \\
1 & 1 & -1 & -1 \\
1 & -1 & 1 & -1 \\
1 & -1 & -1 & 1
\end{array}\right)\left(\begin{array}{c}
w^{0} \\
w^{1} \\
w^{2} \\
w^{3}
\end{array}\right) \\
& ={ }^{t}\left(\zeta^{0} \zeta^{1} \zeta^{2} \zeta^{3}\right)=\zeta, \text { where } \beta=2 \cdot 2^{9} \cdot 2^{N} / \pi \varepsilon .
\end{aligned}
$$

If $\operatorname{Im} w^{\mu}>\varepsilon\left|\operatorname{Re} w^{\mu}\right|$ for $\mu=0, \cdots, 3$, it is easy to see that

$$
\operatorname{Im} \zeta^{0}=\beta|\operatorname{Im} w|>\beta\left|\operatorname{Im} \zeta^{\mu}\right| \text { for every } \mu,
$$$$
\operatorname{Im} \zeta^{0}=\beta|\operatorname{Im} w|>\beta \varepsilon|\operatorname{Re} w|>\varepsilon|\operatorname{Re} \zeta|,
$$

Decompose $\zeta$ into $c\left(\xi+\omega+\left(\rho^{0}, 0\right)\right)$ by taking $\xi^{0}=\left(\operatorname{Im} \zeta^{0}\right) / 2, \omega^{0}=0, \rho^{0}$ $=\left(\operatorname{Im} \zeta^{0}\right) / 2-i \operatorname{Re} \zeta^{0}, \xi^{k}=\operatorname{Re} \zeta^{k}, \omega^{k}=i \operatorname{Im} \zeta^{k}(k=1,2,3)$. Then it is not difficult to verify, using $(8 \cdot 16)-(8 \cdot 18)$, that $\zeta \in \iota\left(\Gamma_{1, \varepsilon}^{(N)}+C_{1}{ }^{(N)}\right)$

Corollary 8.8. For any positive $\varepsilon$, there exists a linear transformation $L_{\varepsilon, k}^{(N)}=\bigotimes_{k} L_{\varepsilon}{ }^{(N)}$ which maps $\left\{w \in \mathbb{C}^{4} ; \operatorname{Im} w^{\mu}>\varepsilon\left|\operatorname{Re} w^{\mu}\right|, \mu=0, \cdots, 3\right\}^{k}$ into $\iota\left(\Gamma_{k, \varepsilon}^{(N)}+C_{k}^{(N)}\right)$.

Proof. If one takes $\beta=2 \cdot 2^{9} \cdot 2^{N} \cdot k / \pi \varepsilon$ and constructs $L_{\varepsilon}{ }^{(N)}$ as above, the corollary immediately follows.

Proposition 8.9. Define for $f \in P_{4 k}$

$$
W_{k}(f)=\int_{r} 4 k W_{k}\left(L_{\varepsilon, k}^{(N)} \underline{w}_{k}\right) f\left(L_{\varepsilon, k}^{(N)} \underline{w}_{k}\right)\left|L_{\varepsilon, k}^{(N)}\right| d \underline{w}_{k},
$$

where $\gamma$ is a path of integration lying in $\{w \in C ; \operatorname{Im} w>\varepsilon|\operatorname{Re} w|\}$ (see $\mathrm{NM}$ II), then $W_{k} \in\left(\mathscr{Q}_{4 k}\right)^{\prime}$. 
Proof. Since $W_{k}\left(\zeta_{k}\right)$ is an infra-exponential holomorphic function, the right hand side of Eq. (8.19) is meaningful. It is sufficient to prove that the right hand side of $(8 \cdot 19)$ is independent of $L_{\varepsilon, k}^{(N)}$ and $\gamma^{4 k}$ as far as it is defined. We rewrite it as follows:

$$
\text { r.h.s. of }(8 \cdot 19)=\int_{r^{\prime 4 k}} W_{k}\left(\underline{\zeta}_{k}\right) f\left(\underline{\zeta}_{k}\right) d \zeta_{k},
$$

where $\gamma^{\prime 4 k}=L_{\varepsilon, k}^{(N)} \gamma^{4 k}$ is a surface contained in $\iota\left(\Gamma_{k, \varepsilon}^{(N)}+C_{k}^{(N)}\right)$. By the Cauchy-Poincaré theorem (see p.198 of Vladimirov [19]), the integral (8.20) is independent of the surface as far as it is defined. This proves the proposition.

Proposition 8.10. The support of the Fourier transform $\widetilde{W}_{k}$ of $W_{k}$ is contained in $\overline{\left(\boldsymbol{R}_{+} \times \boldsymbol{R}^{3}\right)^{k}}$.

Proof. We only prove the proposition for the case $k=1$. Since $\mathscr{Q}_{4}$ is dense in $\underset{\approx}{\left(\boldsymbol{R}_{+} \times \mathbb{R}^{3}\right)}$ (see the Appendix), it is sufficient to show that

$$
|\widetilde{W}(f)| \leqq C_{\varepsilon} \sup _{z \in \Sigma_{\varepsilon}}|f(z)| e^{\varepsilon|z|} \quad \text { for all } f \in \mathscr{L}_{4},
$$

where $\Sigma_{\varepsilon}=\left\{z \in C^{4} ; \operatorname{Re} z^{0}>-\varepsilon,\left|\operatorname{Im} z^{\mu}\right|>\varepsilon\left(1+\left|\operatorname{Re} z^{\mu}\right|\right)\right\} \quad$ is a neighbourhood of $\boldsymbol{R}_{+} \times \boldsymbol{R}^{3}$ in $Q^{4}$. Here and in what follows in the proof of the proposition the suffix 1 indicating $k=1$ is suppressed and $L$ is a short hand notation for $L_{\varepsilon}{ }^{(N)}$.

$$
\begin{aligned}
\widetilde{W}(f) & =\widetilde{W}(\mathscr{I} \overline{\mathscr{L}} f) \\
& =(2 \pi)^{-4} \int_{r^{4}}\left\{W(L w) \int e^{-i(L w) \cdot p} f(p) d p\right\}|L| d w \\
& \left.=(2 \pi)^{-4} \int_{r^{4}}\left\{W(L w) \int e^{-i w \cdot(t L p)} f(p) d p\right\}\left|{ }^{t} L\right| d p\right\} d w \\
& \left.=(2 \pi)^{-4} \int_{r^{4}}\left\{W(L w) \int e^{-i w \cdot p} f\left({ }^{t} L^{-1} p\right) d p\right\}\right\} d w .
\end{aligned}
$$

We now introduce quadrants centered at $x=-\delta, 0<\delta<\varepsilon$, as follows:

$$
\begin{aligned}
(* * * *) & =\left\{\left(x^{0}, x^{1}, x^{2}, x^{3}\right) \in \boldsymbol{R}^{4} ; *=+ \text { if } x^{\mu}>-\delta,\right. \\
* & \left.=- \text { if } x^{\mu}<-\delta \text { for each } \mu\right\} .
\end{aligned}
$$


Since

$$
{ }^{t} L^{-1}=\frac{1}{4}\left(\begin{array}{rrrr}
1 / \beta & 1 / \beta & 1 / \beta & 1 / \beta \\
1 & 1 & -1 & -1 \\
1 & -1 & 1 & -1 \\
1 & -1 & -1 & 1
\end{array}\right)
$$

${ }^{t} L^{-1}(++++)$ is contained in $(-\delta / \beta, \infty) \times \boldsymbol{R}^{3}$. Define

$$
g_{* * * *}(w)=\int_{(* * * *)} e^{-i w \cdot p} f\left({ }^{t} L^{-1} p\right) d p
$$

then it is easily seen that $g_{++++}(w)$ is a rapidly decreasing holomorphic function and satisfies the estimate

$$
\sup _{w \in U_{m}}\left|g_{++++}(w) e^{|w| / m}\right| \leqq C_{\varepsilon} \sup _{z \in \Sigma_{\varepsilon}}\left|f(z) e^{\varepsilon|z|}\right| \quad \text { for } m>1 / \varepsilon
$$

As for other quadrants, for example $(-+++), g_{-+++}(w)$ belongs to $\mathscr{L}_{4}$; moreover it is analytic in $\left\{\operatorname{Im} w^{0}>0\right\}$ and satisfies there the estimate

$$
\mid g_{-+++}(w) \leqq C e^{-\delta\left(\operatorname{Im} w^{0}\right)} .
$$

Therefore, by introducing the path $\gamma^{\omega}=\{w ; w-i \omega \in \gamma\}$, we obtain

$$
(2 \pi)^{-4} \int_{r^{4}} W(L w) g_{-+++}(w) d w=\lim _{\omega \rightarrow \infty} \int_{r^{3} \times r^{\omega}} W(L w) g_{-+++}(w) d w=0 .
$$

Thus we have

$$
\begin{aligned}
|\widetilde{W}(f)| & =\left|\int_{r^{4}} W(L w)\left\{\sum_{(* * * *)} g_{* * * *}(w)\right\} d w\right| \\
& =\left|\int_{r^{4}} W(L w) g_{++++}(w) d w\right| \leqq C_{\varepsilon} \sup _{z \in \Sigma_{\varepsilon}}|f(z)| e^{\varepsilon|z|},
\end{aligned}
$$

which completes the proof.

Remark. From the Euclidean convariance (E1) follows the relativistic invariance of $\widetilde{W}_{k}$. Therefore the support of $\widetilde{W}_{k}$ is contained in $\overline{V_{+}{ }^{k}}$.

Proposition 8.11. $S_{k}\left(\underline{\xi}_{k}\right)=W_{k}\left(\ell \underline{\xi_{k}}\right)=\widetilde{W}_{k}\left(e^{\left.((\cdot, \ell \underline{\xi}))_{k}\right)}\right.$ for $\underline{\xi}_{k}{ }^{0} \in \boldsymbol{R}_{+}{ }^{k}$ 。

Proof. By Proposition 3.13 of NM II, from the equality 


$$
\begin{array}{r}
\int_{r^{4 k}} W_{k}\left(L_{\varepsilon, k}^{(N)} \underline{w}_{k}\right) f\left(L_{\varepsilon, k}^{\left.(N) \underline{w}_{k}\right)}\right)\left|L_{\varepsilon, k}^{(N)}\right| d w_{k}=\widetilde{W}_{k}(\mathscr{F} f) \\
=\int_{r^{4 k}} \widetilde{W}_{k}\left(e^{i\left(\left(\cdot, L_{\varepsilon, k}^{(N)} w\right)\right)_{k}}\right) f\left(L_{\varepsilon, k}^{(N)} \underline{w}_{k}\right)\left|L_{\varepsilon, k}^{(N)}\right| d \underline{w}_{k}
\end{array}
$$

valid for $f \in \mathscr{Q}_{4 k}, \varepsilon>0$ and positive $N$, there follows $W_{k}\left(L_{\varepsilon, k}^{(N)} \underline{w}_{k}\right)$ $=\widetilde{W}_{k}\left(\exp \left\{i\left(\left(\cdot, L_{\varepsilon, k}^{(N)} w\right)\right)_{k}\right\}\right)$ for $\operatorname{Im} w_{i}{ }^{\mu}>\varepsilon\left|\operatorname{Re} w_{i}{ }^{\mu}\right|, i=1, \cdots, k, \mu, \cdots, 3$, and all $\varepsilon>0$ and $N$. This implies $W_{k}\left(\iota \xi_{k}\right)=\widetilde{W}_{k}\left(\exp \left\{i(\cdot(\cdot, \xi \xi))_{k}\right\}\right)$ for $\xi_{k}{ }^{0} \in \boldsymbol{R}_{+}{ }^{k}$.

Proof of Thoerem 8.1. Define $\mathfrak{W}_{n}\left(x_{n}\right)=W_{n-1}\left(\xi_{n-1}\right)$. The hyperfunction property (R0) is obvious from Lemma 8.6. The positivity condition (R2) follows from Lemma 8.4 and the fact that the Wightman function $W_{n-1}\left(\xi_{n-1}\right)$ can be obtained as a boundary value of the analytic continuation of the Schwinger function $S_{n-1}$. (E4) shows that for any vector $\Phi, \Psi \in \mathcal{K}$ we have $\lim _{\lambda \rightarrow \infty}\left\langle\Phi, U_{s}(\lambda a) \Psi\right\rangle=\langle\Phi, \Omega\rangle\langle\Omega, \Psi\rangle$, which implies the cluster property (R4). The relativistic covariance (R1) and the local commutativity (R3) are proved by the same arguments as used by Osterwalder and Schrader [13]. (R5) has already mentioned after the proof of Proposition 8.10. Proposition 8.11 implies that the corresponding Schwinger functions coincide with the Euclidean Green's functions given initially. Uniqueness follows from Proposition 7.3. The proof of Theorem 8.1 is thus completed.

\section{Appendix $\mathscr{Q}_{* *}$ is dense in $\mathcal{O}(K)$}

Here we wish to prove the statement: Let $K$ be a compact subset

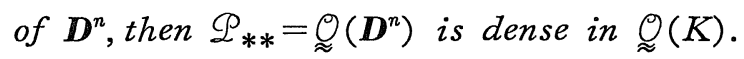

The proof goes on in a fashion similar to that in Theorem 2.2.1 of Kawai [10], if we can construct a sequence of subsets $\left\{\Omega_{p}\right\}$ possessing the following properties:

(a) $U_{p}^{n} \supset \Omega_{p} \supseteq K$ and $\Omega_{p}$ 's tend decreasingly to $K$, where $U_{p}$ is given by $(3 \cdot 1)$.

(b) For any $p$ and any $T\left(\mathbb{E} \Omega_{p}\right)$ there exist an open set $V$ and a function $\theta(z)$ strictly plurisubharmonic in $U_{p}{ }^{n}$ such that (i) $T \subset V \Subset \Omega_{p}$. 
(ii) $\theta(z)<0$ on $T \subset \mathbb{C}^{n}$.

(iii) $\theta(z)>0$ near $\partial V \subset \mathbb{C}^{n}$.

(iv) $\sup _{L \cap C^{n}} \theta(z) \leqq M_{L}<\infty$ for any $L \subset \Omega_{p}$.

Construction of $\left\{\Omega_{p}\right\}$ : We shall say that $\Omega$ is of type $(E)$ if $\Omega$ $=\bigcap_{l=1}^{\infty} V^{l}$, where

$$
V^{l}=\left\{z \in \mathbb{C}^{n} ;\left|f_{l}(z)\right|<1, d_{l}\left|\operatorname{Im} z_{j}\right|^{2}-\left|\operatorname{Re} z_{j}\right|^{2}<1,1 \leqq j \leqq n\right\} .
$$

Here $f_{l}(z)=c_{l} \exp \left\{-\sum_{j}\left(z_{j}-a_{j}{ }^{(l)}\right)^{2}\right\}, c_{l} \in \mathbb{R}$ and $a^{(l)} \in \mathbb{R}^{n}$. Since $K$ is a compact set in $\mathbb{D}^{n}$, it is clear that $K$ can be approximated by a decreasing sequence of $\Omega_{p}$ of type $(E)$.

We are going to construct $V$ and $\theta(z)$ having the required properties for any $T \Subset \Omega_{p}$. From now on we suppress index $p$ of $\Omega_{p}$. By the definition of $T$ one can find $\left\{G_{k}\right\}$ such that $T \in \bigcup_{k=1}^{m} G_{k}$, where $G_{k}$ is a relatively compact set in $\mathbb{C}^{n}$ or an open convex cone in $\mathbb{C}^{n}$ and $G_{k} \Subset \Omega$. Then by taking a suitable set $S$ of type $(E)$, we have $T \subset S$ and $\mathrm{S}$ $\cap\{|\operatorname{Re} z|>M\} \Subset[\Omega \cap\{|\operatorname{Re} z|>M\}]$. On the other hand, recalling that $\Omega=\bigcap_{l} V^{l}$ by the definition of $\Omega$ we have $T \cap\{|\operatorname{Re} z|<M+1\} \Subset V_{\varepsilon}^{l \pm \ldots \pm}$ $\cap\{|\operatorname{Re} z|<M+1\}$ for sufficiently small $\varepsilon$, where $V_{\varepsilon}^{l \pm \ldots \pm}$ is a translation of $V$ parallel to the coordinate axis by $\pm \varepsilon$. Thus, taking $\varepsilon$ sufficiently small, we have $T \Subset V_{\varepsilon}^{l_{ \pm \ldots} \ldots \pm}$. We define $V=S \cap\left(\cap V_{\varepsilon}^{l_{ \pm \cdots \pm}}\right)$.

By The above construction $V$ can be represented as $\cap_{l} V^{2}$. As is clear from the method of construction of $\Omega$ and $V$, we may assume $d_{l}=d$ without loss of generality.

On setting $\phi(z)=\max _{j}\left\{d\left|\operatorname{Im} z_{j}\right|^{2}-\left|\operatorname{Re} z_{j}\right|^{2}-1\right\}, \sigma(z)=\sup _{l} \log \left|f_{l}(z)\right|$ and $\chi(x)=\max \{\phi(z), \sigma(z)\}$, we define

$$
\theta(z)=\chi(z) * \rho_{\varepsilon}+\varepsilon\left[1+\log \left(1+|z|^{2}\right)\right]
$$

where $\rho_{\varepsilon}$ is a molifier in $\boldsymbol{R}^{2 n}$. Then $\theta(z)$ thus defined is strictly plurisubharmonic and $\theta(z)<0$ on $T$, and $\theta(z)>0$ near $\partial V$ for sufficiently small $\varepsilon>0$, thus all the required properties having been satisfied.

\section{References}

[1] Constantinescu, F., Analytic properties of nonstrictly localizable fields, J. Math. Phys. 12 (1971), 293-298.

[2] Constantinescu, F. and Thalheimer, W., Euclidean Green's functions for Jaffe fields, Comm. Math. Phys. 38 (1974), 299-316.

[3] Gel'fand, I. M. and Shilov, G. E., Generalized functions Vol. 2, (Academic Press, 
New York-London, 1968).

[4] Glaser, V., On the equivalence of the Euclidean and Wightman formulation of field theory, Comm. Math. Phys. 37 (1974), 257-272.

[5] Grothendieck, A., Espaces vectoriels topologiques, (Soc. Math. São Paulo, 1964).

[6] Hörmander, L., $L^{2}$ estimates and existence theorems for the $\bar{\partial}$ operator, Acta Math. 113 (1965), 89-152.

[7] Ito, Y. and Nagamachi, S., On the theory of vector valued Fourier hyperfunctions, J. Math. Tokushima Univ. 9 (1975), 1-33.

[8] Jaffe, A., High-energy behavior in quantum field theory I. Strictly localizable fields, Phys. Rev. 158 (1967), 1454-1461.

[9] Jost, R., The general theory of quantized fields, (Amer. Math. Soc., Providence, 1965).

[10] Kawai, T., On the theory of Fourier hyperfunctions and its applications to partial differential equations with constant coefficients, J. Fac. Sci. Univ. Tokyo IA 17, (1970), 467-517.

[11] Nagamachi, S. and Mugibayashi, N., Hyperfunction quantum fidld theory, Comm. Math. Phys. 46 (1976), 119-134.

[12] Nagamachi, S. and Mugibayashi, N., Hyperfunction xuantum field theory II. Euclidean Green's functions, Comm. Math. Phys. 49 (1976), 257-275.

[13] Osterwalder, K. and Schrader, R., Axioms for Euclidean Green's functions, Comm. Math. Phys. 31 (1973), 83-112.

[14] Osterwalder, K. and Schrader, R., Axioms for Euclidean Green's functions II, Comm. Math. Phys. 42 (1975), 281-305.

[15] Sabad, E. and Lomsadze, Yu. M., Abstract axiomatic approaches in the quantum field theory I, Kiev Preprint ITP-75-94E (1975) and references quoted therein.

[16] Schapira, P., Théorie des hyperfunctions, Lecture Notes in Math. No. 126 (Springer, Berlin-Heidelberg-New York, 1970).

[17] Streater, R. and Wightman, A. S., PCT, spin \& statistics, and all that, (Benjamin, New York-Amsterdam, 1964).

[18] Treves, F., Topological vector spaces, distributions, and kernels, (Academic Press, New York-London, 1967).

[19] Vladimirov, V. S., Methods of the theory of functions of many complex variables, (MIT Press, Cambridge, 1966). 
